\title{
CONTINUATION THEOREMS FOR PERIODIC PERTURBATIONS OF AUTONOMOUS SYSTEMS
}

\author{
ANNA CAPIETTO, JEAN MAWHIN AND FABIO ZANOLIN
}

\begin{abstract}
It is first shown in this paper that, whenever it exists, the coincidence degree of the left-hand member of an autonomous differential equation

$$
x^{\prime}-g(x)=0,
$$

in the space of periodic functions with fixed period $\omega$, can be computed in terms of the Brouwer degree of $g$. This result provides efficient continuation theorems specially for $\omega$-periodic perturbations of autonomous systems. Extensions to differential equations in flow-invariant ENR's are also given.
\end{abstract}

\section{INTRODUCTION}

In this paper we are concerned with the periodic boundary value problem (BVP)

$$
\begin{aligned}
& x^{\prime}=F(t, x), \\
& x(0)=x(\omega),
\end{aligned}
$$

where $F:[0, \omega] \times \mathbf{R}^{m} \rightarrow \mathbf{R}^{m}$ is a Carathéodory function $(\omega>0)$. We recall that if $F: \mathbf{R} \times \mathbf{R}^{m} \rightarrow \mathbf{R}^{m}$ is $\omega$-periodic in the first variable, then any solution of (1.1)-(1.2) can be extended to a classical (i.e. absolutely continuous) $\omega$ periodic solution of (1.1) defined on the whole real line. Accordingly, without overindulging in terminology, we call in what follows $\omega$-periodic any solution of (1.1) satisfying (1.2).

The periodic BVP plays a central role in the theory of ODEs for its significance in several applications (see [30, 58, 59]). Many authors have treated problem (1.1)-(1.2) by means of topological methods; in such a framework, continuation theorems turn out to be specially suitable for the existence problem. Basically, the "continuation" is performed through an admissible homotopy carrying the given problem to a simpler one. This simpler one may be an autonomous equation whose $\omega$-periodic solutions consist in an odd number of nondegenerate equilibria, like in Stoppelli's pioneering work [63], or a linear equation having only the trivial $\omega$-periodic solution (see e.g. [43], references for Theorem IV.5). By the fundamental properties of topological degree theory, such an approach will only succeed in problems having an odd degree.

Received by the editors May 11, 1989 and, in revised form, March 5, 1990.

1980 Mathematics Subject Classification (1985 Revision). Primary 34C25, 61F22, 49H15; Secondary $58 \mathrm{M} 25$.

Work performed under the auspices of G.N.A.F.A.-C.N.R., Italy. 
Two different methods have been developed by M. A. Krasnosel'skii [30] and J. Mawhin [43] which carry the problem to simpler ones induced by some associated autonomous vector field. On the one side, in [30, Chapter 2] a homotopy through the trajectories of (1.1) is considered and the existence of a fixed point of the Poincare map is proved via a degree theoretic assumption on the autonomous function $F(0, \cdot): \mathbf{R}^{m} \rightarrow \mathbf{R}^{m}$. In this case, the admissibility of the homotopy is guaranteed by the so-called $\omega$-irreversibility condition. On the other hand, in [43] system (1.1) is embedded into the parametrized family of equations $x^{\prime}=\lambda f(t, x ; \lambda), \lambda \in(0,1)$, with $f(t, x ; 1)=F(t, x)$ and the solvability of $(1.1)-(1.2)$ is ensured via a degree theoretic assumption on the autonomous averaged vector field $\bar{f}_{0}: z \mapsto(1 / \omega) \int_{0}^{\omega} f(s, z ; 0) d s$. In this case another transversality condition is required for the admissibility of the homotopy.

Both Krasnosel'skii's and Mawhin's theorems have found useful applications in the literature (see for instance the references in $[2,22,31,43,52,59,60]$. For other different but related results see [35, 57, 62].

An important situation which occurs in several applications corresponds to the case when the nonautonomous field $F(t, x)$ splits as

$$
F(t, x):=g(x)+e(t, x),
$$

where $e(\cdot, \cdot)$ satisfies suitable growth conditions (e.g. is bounded).

In such a situation, it is natural to choose the homotopy field $f(t, x ; \lambda):=$ $g(x)+\lambda e(t, x), \lambda \in[0,1]$; however, none of the previously quoted continuation theorems can be directly applied.

The aim of our work is to provide a new continuation result for (1.1)-(1.2) which is particularly suitable for dealing with nonlinearities like (1.3). To do this, we assume that

$$
F(t, x):=f(t, x ; 1),
$$

where $f=f(t, x ; \lambda):[0, \omega] \times \mathbf{R}^{m} \times[0,1] \rightarrow \mathbf{R}^{m}$ is a Carathéodory function such that for $\lambda=0$ the map $f$ is autonomous, i.e. there is a continuous function $f_{0}: \mathbf{R}^{m} \rightarrow \mathbf{R}^{m}$ such that

$$
f_{0}(x)=f(t, x ; 0),
$$

for almost all $t \in[0, \omega]$ and all $x \in \mathbf{R}^{m}$. A crucial point in the proof of continuation theorems is to show that the "degree" (or, in more general situations, the fixed point index) of (suitable) operators defined in function spaces is equal to the Brouwer degree of a (corresponding) autonomous map which takes values in $\mathbf{R}^{m}$. For example, in [43, Theorem IV.13] the continuation technique is associated to the homotopy

$$
(\lambda, x) \mapsto(1-\lambda) \frac{1}{\omega} \int_{0}^{\omega} f(s, x(s) ; \lambda) d s+\lambda f(s, x ; \lambda)
$$

leading for $\lambda=0$ to the integro-differential system

$$
x^{\prime}-\frac{1}{\omega} \int_{0}^{\omega} f(s, x(s) ; 0) d s=0,
$$

whose $\omega$-periodic solutions are constant and given by the zeros of $\bar{f}_{0}$. In [43, Lemma VI.1], the continuation is made through the homotopy

$$
(\lambda, x) \mapsto(1-\lambda) \nabla V(x)+\lambda F(t, x),
$$


for some $C^{1}$ function $V: \mathbf{R}^{m} \rightarrow \mathbf{R}$. This gives, for $\lambda=0$, the gradient system

$$
x^{\prime}-\nabla V(x)=0 \text {, }
$$

whose $\omega$-periodic solutions are given by the zeros of $\nabla V$. In both cases, it is easy to compute the (coincidence) degree (or, in more general situations, the fixed point index), in a suitable space of $\omega$-periodic functions, of the operator associated to the left-hand member of (1.4) (resp. (1.5)), in terms of the Brouwer degree in $\mathbf{R}^{m}$ of the mapping $\bar{f}_{0}$ (resp. $\nabla V$ ). For a homotopy

$$
(\lambda, x) \mapsto f(t, x ; \lambda),
$$

connecting $F(t, x)$ (for $\lambda=1$ ) to a general autonomous field $f_{0}(x)$ for $\lambda=0$, the computation of the (coincidence) degree of the operator defined by the lefthand member of the equation

$$
x^{\prime}-f_{0}(x)=0
$$

is made more difficult by the presence of possible nonconstant closed orbits with period less than $\omega$. Theorem 1 in $\S I I .1$ shows however that a similar connection still holds between the coincidence degree and the Brouwer degree of $f_{0}$. To obtain this result, we use an "approximation" procedure for the map $f_{0}$ based on the Kupka-Smale theorem [7, 54]. The Kupka-Smale theorem ensures the existence of a sequence $\left(\varphi_{k}\right)$ of $C^{1}$-functions, $\left(\varphi_{k}\right) \rightarrow f_{0}$, such that for each $\sigma>0$, for every compact subset $K$ of $\mathbf{R}^{m}$ and for all $k \in \mathbf{N}$, system $x^{\prime}=\varphi_{k}(x)$ has finitely many rest points or closed orbits with period less or equal than $\sigma$ which are contained in $K$. By means of this result, and using the additivity/excision property of the coincidence degree, we can perform admissible homotopies which lead to the conclusion.

Notice that Theorem 1 can be viewed as a contribution to the computation of coincidence degree in spaces of periodic functions.

Now, there are various ways to express the problem of $\omega$-periodic solutions of (1.6) in terms of fixed points of operators defined in the space of continuous functions on $[0, \omega]$. Using duality theorems developed in [31, Chapter III] and [43, Chapter III] and Theorem 1, one can also express the Leray-Schauder degree of these operators in terms of the Brouwer degree of $f_{0}$, and this is the object of Corollary 1. Finally, similar results also hold which connect the Brouwer degree of the Poincaré-Andronov operator for $\omega$-periodic solutions to the Brouwer dezree of $f_{0}$ (Corollary 2 ).

In $\S I I .2$ we apply Theorem 1 to the proof of Theorem 2, a continuation theorem for the periodic solutions to (1.1)-(1.2) when the family of parametrized equations is

$$
x^{\prime}=f(t, x ; \lambda), \quad \lambda \in[0,1],
$$

with $f(t, x ; 1)=F(t, x)$ and $f(t, x ; 0)=f_{0}(x)$, and it is assumed that the Brouwer degree of the map $f_{0}$ is different from zero. Theorem 3 is an extension of Theorem 2 to the case where the phase space $\mathbf{R}^{m}$ is replaced by a closed convex set with nonempty interior. Theorem 4 is a continuation theorem similar to Theorem 2 but expressed in terms of the Poincaré-Andronov operator.

Although $\S I I$ already provides examples comparing the applicability of Theorem 2 to that of other continuation results, more applications are given in $\S I I I$ for the important case where $F$ splits as

$$
F(t, x)=g(x)+e(t, x),
$$


where $e(t, x)$ satisfies suitable growth conditions and the performed homotopy is

$$
f(t, x ; \lambda)=g(x)+\lambda e(t, x) .
$$

A first class of results are of perturbational type, i.e. require $|e|_{\infty}$ to be sufficiently small. In this case, the assumptions upon $g$ are rather mild and the results generalize in various ways earlier contributions of Amel'kin-GaishunLadis [1], Berstein-Halanay [3], Cronin [9, 10, 11], Gomory [23], Halanay [25, 27], Hale-Somolinos [28], Lando [32, 33], Pliss [56], Srzednicki [62] and Ward [66]. A second class of results, of global type, deals with the case where $g$ is positively homogeneous of some order and the corresponding results improve in various directions earlier contributions of Dancer [13], Fonda-Habets [17], Fonda-Zanolin [18], Fučik [19], Krasnosel'skii-Zabreiko [31], Lazer-McKenna [36], Lasota [34] and Muhamadiev [47, 48].

In $\S I V$, an extension of Theorem 2 to flow-invariant Euclidean Neighbourhood Retracts is performed (Theorem 5). We recall that a metric space $C$ is an ANR (Absolute Neighbourhood Retract) if and only if it is homeomorphic to a neighbourhood retract of a Banach space $Y$. If $Y$ is finite dimensional, we say that $C$ is an ENR (Euclidean Neighbourhood Retract). In such a general framework we use (continuous vector fields and) the fixed point index of compact operators defined on the space of continuous functions which take values in the given ENR. Moreover, Theorem 5 enables us to deal with some cases when the phase space is not the whole $\mathbf{R}^{m}$ but e.g. a regular manifold, a closed convex set or a conical shell. Beside a flow-invariance condition for the given ENR, we introduce the so-called "property (A)", which ensures that the ENR we work with is invariant not only for the flow induced by $x^{\prime}=f_{0}(x)$ but for the flow induced by $x^{\prime}=\varphi_{k}(x)$ as well, where (according to the Kupka-Smale's theorem) $\left(\varphi_{k}\right)$ is the sequence of functions approximating $f_{0}$. We point out that property (A) holds (obviously) true when the phase space is $\mathbf{R}^{m}$ itself (as in $\S \S I I$ and III); moreover, it is always satisfied in the cases we quoted above (e.g. regular manifolds and closed convex sets) as well. The proof of Theorem 5 is carried out by embedding (1.1)-(1.2) in a functional-analytic framework which is inspired by the study of the Poincare map (like in [6]); on the other hand, we use, instead of the Brouwer degree, the "index of rest points".

We end this section with a list of notations. The $m$-dimensional real euclidean space $\mathbf{R}^{m}$ is endowed with the usual scalar product $(\cdot \mid \cdot)^{\frac{1}{2}}$, norm $|\cdot|$ and distance $d(\cdot, \cdot)$. Given two subsets $C_{1}, C_{2}$ of $\mathbf{R}^{m}$, we denote by

$$
d\left(C_{1}, C_{2}\right):=\inf \left\{|a-b|: a \in C_{1}, b \in C_{2}\right\},
$$

the distance between the sets $C_{1}$ and $C_{2}, \mathbf{R}_{+}$is the set of nonnegative reals. Given $x_{0} \in \mathbf{R}^{m}$ and $R>0$, we denote by $B\left(x_{0}, R\right)$ (resp., $B\left[x_{0}, R\right]$ ) the open (resp., closed) ball of center $x_{0}$ and radius $R$. Given any metric space $W$, for $A \subset B \subset W$ by $\operatorname{int}_{B} A, \mathrm{fr}_{B} A, \mathrm{cl}_{B} A$ we mean, respectively, the interior, boundary and closure of the set $A$ relatively to $B$. We omit the subscript when $B=W=\mathbf{R}^{m}$. If $W$ is a normed space, $|\cdot|_{W}$ denotes its norm and $I_{W}$ the identity operator in $W$. As a usual convention, the subscript is omitted for $W=\mathbf{R}^{m}$.

For a closed set $S \subset \mathbf{R}^{m}$, by

$$
T(z ; S):=\left\{v \in \mathbf{R}^{m}: \liminf _{h \rightarrow 0^{+}} d(z+h v, S) / h=0\right\},
$$


we mean the (Bouligand) tangent cone to $S$ at $z$, while, for a closed convex set $K \subset \mathbf{R}^{m}$ we denote by $N(z, K)$ the set of (nonzero) outer normals to $K$ at $z \in \operatorname{fr} K$.

Let $\omega>0$ be a fixed constant. For a function $y:[0, \omega] \rightarrow \mathbf{R}^{m}$, we use the respective norms $|y|_{\infty}:=\sup \{|y(t)|: t \in[0, \omega]\},|y|_{1}:=\int_{0}^{\omega}|y(s)| d s$ in the associated function spaces and we set

$$
\bar{y}:=\frac{1}{\omega} \int_{0}^{\omega} y(s) d s
$$

Finally, if $C$ is an ANR and $\psi: \operatorname{cl}_{C} U \rightarrow C$ (with $U$ bounded and open relatively to $C$ ) is a compact map with $\psi(x) \neq x$ for $x \in \operatorname{fr}_{C} U$, we denote by $i_{C}(\psi, U)$ the corresponding fixed point index (see [24, 50]). By $d_{B}$ and deg we mean, respectively, the usual Brouwer degree in $\mathbf{R}^{m}$ and the Leray-Schauder degree in a normed vector space.

\section{THE MAIN RESULT}

We deal with the periodic boundary value problem:

$$
\begin{aligned}
& x^{\prime}=F(t, x), \\
& x(0)=x(\omega),
\end{aligned}
$$

where

$$
F(t, x):=f(t, x ; 1)
$$

and $f=f(t, x ; \lambda):[0, \omega] \times \mathbf{R}^{m} \times[0,1] \rightarrow \mathbf{R}^{m}$ satisfies the Carathéodory conditions, i.e. $f(\cdot, x ; \lambda)$ is (Lebesgue) measurable for each $(x, \lambda), f(t, \cdot ; \cdot)$ is continuous for a.e. $t$ and, for each $r>0$, there exists $\beta_{r} \in L^{1}([0, \omega], \mathbf{R})$ such that $|f(t, x ; \lambda)| \leq \beta_{r}(t)$ holds for a.e. $t \in[0, \omega]$ and all $|x| \leq r, \lambda \in[0,1]$. Accordingly, solutions for $x^{\prime}=f(t, x ; \lambda)$ are intended in the generalized (i.e. Carathéodory) sense. With small abuse in the terminology, we call $\omega$-periodic any solution satisfying the boundary condition (2.2).

As we mentioned in the introduction, we assume that for $\lambda=0$ the map $f$ is autonomous, i.e. there exists a continuous function $f_{0}: \mathbf{R}^{m} \rightarrow \mathbf{R}^{m}$ such that

$$
f_{0}(x):=f(t, x ; 0)
$$

for almost every $t \in[0, \omega]$ and each $x \in \mathbf{R}^{m}$. A particular but significant case in which such a situation occurs is when $f$ splits as

$$
f(t, x ; \lambda)=f_{0}(x)+\lambda e(t, x ; \lambda) ;
$$

this is examined in detail in $\S$ III.

The proof of continuation results for problem (2.1)-(2.2) is based, essentially, on the homotopy invariance of the topological degree and on estimates for the degree of some operators associated to system

$$
x^{\prime}=f_{0}(x) \text {. }
$$

This second goal is achieved showing that, under certain circumstances, it is sufficient to evaluate the (finite dimensional) Brouwer degree of the vector field $f_{0}$. In the first part of this section, we prove some results in which the above programme is developed for various operators related to (2.5).

In what follows, the (real) Banach spaces $Z:=L^{1}\left([0, \omega], \mathbf{R}^{m}\right), \quad Y:=$ $C\left([0, \omega], \mathbf{R}^{m}\right)$ and $X:=\{x \in Y: x(0)=x(\omega)\}$, with their usual norms, are considered. Notice that points of $\mathbf{R}^{m}$ are identified with constant functions. 
II.1. Estimates for the degree. For the first result, we recall some basic facts from coincidence degree theory, borrowing notation and terminology from [43]. We define $L: \operatorname{dom} L \subset X \rightarrow Z, L x=x^{\prime}$, a Fredholm mapping of index zero, with $\operatorname{dom} L=\{x \in X: x(\cdot)$ is absolutely continuous $\}$.

Let $M_{0}$ be the Nemitzky operator from $X$ to $Z$ induced by the map $f_{0}$, i.e. $M_{0}: x(\cdot) \mapsto f_{0}(x(\cdot))$.

In this situation, problem (2.5)-(2.2) can be transformed into the equivalent coincidence equation:

$$
L x=M_{0} x, \quad x \in \operatorname{dom} L .
$$

If we introduce the linear projectors $Q: Z \rightarrow$ coker $L \subset Z, Q z:=\bar{z}=$ $(1 / \omega) \int_{0}^{\omega} z(s) d s$ and $P:=\left.Q\right|_{X}: X \rightarrow \operatorname{ker} L \subset X$ and we denote by $K_{P, Q}: Z \rightarrow$ ker $P \cap \operatorname{dom} L$ the generalized inverse of $L$, then equation (2.6) is equivalent to

$$
x=R_{0}(x):=P x+K_{P, Q} M_{0} x+J Q M_{0} x=x-\left(J Q+K_{P, Q}\right)\left(L-M_{0}\right) x,
$$

where $J: \operatorname{Im} Q=\mathbf{R}^{m} \rightarrow \operatorname{ker} L=\mathbf{R}^{m}$ is a linear isomorphism, so that $I-R_{0}=$ $T\left(L-M_{0}\right)$, for some linear isomorphism $T$ (see $\left.[43,44,55,65]\right)$.

In the sequel, for simplicity, we take $J:=I$ (the identity in $\mathbf{R}^{m}$ ).

Let $\Omega \subset X$ be bounded and open (relatively to $X$ ).

It is a standard fact to check that $R_{0}: \mathrm{cl}_{X} \Omega \rightarrow X$ is compact. Therefore, the coincidence degree of $L$ and $M_{0}$ in $\Omega$ is defined by

$$
D_{L}\left(L-M_{0}, \Omega\right):=\operatorname{deg}\left(I_{X}-R_{0}, \Omega, 0\right),
$$

provided that

$$
L x \neq M_{0} x \quad \text { for all } \quad x \in \mathrm{fr}_{X} \Omega \cap \operatorname{dom} L .
$$

From [43, p. 19] we know that the definition of the coincidence degree is independent of the projectors $P$ and $Q$.

We note that a similar framework may be introduced by a different choice of the function spaces. In particular, the use of $Z:=L^{1}\left([0, \omega], \mathbf{R}^{m}\right)$ is not necessary at this point. However, such a choice is convenient as we deal later with nonautonomous nonlinearities satisfying only the Carathéodory conditions (see [43, Chapter VI]).

The following theorem, which is crucial for the proof of Theorem 2, may be considered of some independent interest as a contribution to the coincidence degree theory.

Theorem 1. Assume that there is no $x(\cdot) \in \mathrm{fr}_{X} \Omega$ such that $x^{\prime}=f_{0}(x)$. Then,

$$
D_{L}\left(L-M_{0}, \Omega\right)=(-1)^{m} d_{B}\left(f_{0}, \Omega \cap \mathbf{R}^{m}, 0\right) .
$$

Proof. First of all, we observe that, as $\Omega$ is bounded, there is a constant $R>0$ such that $|x|_{\infty}<R$, for every $x \in \operatorname{cl}_{X} \Omega$. Furthermore, we point out that the assumption is equivalent to

$$
L x \neq M_{0} x,
$$

for all $x \in \operatorname{dom} L \cap \operatorname{fr}_{X} \Omega$; therefore, the coincidence degree $D_{L}\left(L-M_{0}, \Omega\right)$ is well defined.

The proof is performed by means of a corollary of the Kupka-Smale's theorem [7, p. 68]; this result ensures the existence of a sequence of $C^{1}$-functions $\left(\varphi_{k}\right), \varphi_{k}: \mathbf{R}^{m} \rightarrow \mathbf{R}^{m}$, such that:

(a) $\left(\varphi_{k}\right) \rightarrow f_{0}$ uniformly on compact sets; 
(b) for every compact subset $K$ of $\mathbf{R}^{m}$ and for all $k \in \mathbf{N}$, system

$$
x^{\prime}=\varphi_{k}(x)
$$

has finitely many singular orbits (i.e., rest points and closed orbits) with minimal period in $[0, \omega+1]$ which are contained in $K$.

Let $N^{k, \mu}$ be the Nemitzky operator induced by the functions $x \mapsto \mu f_{0}(x)+$ $(1-\mu) \varphi_{k}(x), \mu \in[0,1]$. We claim that there is $k_{0}>0$ such that, for all $k \geq k_{0}$ and for all $\mu \in[0,1]$

$$
L x \neq N^{k, \mu} x \quad \text { for all } x \in \operatorname{dom} L \cap \mathrm{fr}_{X} \Omega .
$$

This fact will imply, in particular, that

$$
\varphi_{k}(z) \neq 0 \quad \text { for all } z \in \mathrm{fr}_{X} \Omega \cap \mathbf{R}^{m}, k \geq k_{0} .
$$

Then, a classical compactness argument ensures that, for any $k \geq k_{0}$, there is $\delta_{1}=\delta_{1}(k)$ such that

$$
\varphi_{k}(y) \neq 0 \quad \text { for all } y \in B\left(\mathrm{fr}_{X} \Omega \cap \mathbf{R}^{m}, \delta_{1}\right) .
$$

To obtain (2.9), it is sufficient to observe that the sequence of operators $N^{k, \mu}$ converges, as $k \rightarrow+\infty$, to $M_{0}$ in $Z$ uniformly on $\mathrm{cl}_{X} \Omega \times[0,1]$ and that, by (2.8),

$$
\inf \left\{\left|\left(L-M_{0}\right) x\right|_{Z}: x \in \operatorname{dom} L \cap \mathrm{fr}_{X} \Omega\right\}>0 .
$$

Hence, the claim is proved and, using the homotopy property of the coincidence degree (see [22, Theorem III.2]), we can write

$$
D_{L}\left(L-M_{0}, \Omega\right)=D_{L}\left(L-N^{k, 1}, \Omega\right)=D_{L}\left(L-N^{k, 0}, \Omega\right),
$$

for every $k \geq k_{0}$ and, in particular,

$$
d_{B}\left(f_{0}, \Omega \cap \mathbf{R}^{m}, 0\right)=d_{B}\left(\varphi_{k}, \Omega \cap \mathbf{R}^{m}, 0\right) .
$$

Let us fix $k^{*} \geq k_{0}$. For brevity, we set

$$
\varphi:=\varphi_{k^{*}}, \quad N_{\varphi}:=N^{k^{*}, 0}, \quad \delta_{1}:=\delta_{1}\left(k^{*}\right) .
$$

Consider the singular orbits (i.e. rest points and closed orbits) with minimal period in $[0, \omega+1]$ of the system

$$
x^{\prime}=\varphi(x) .
$$

By the Kupka-Smale theorem, there exist finitely many such orbits which are contained in $B(0, R)$. We denote these orbits by $S_{1}, \ldots, S_{n}$. They are mutually disjoint. Pick, for each $i=1, \ldots, n$, a point $z_{i} \in S_{i}$. Then, $z_{i}$ is a periodic point (possibly a rest point). We can assume that $z_{i}$ is a rest point for $1 \leq i \leq p \quad(p \geq 0$ an integer $)$ and a periodic point for $p+1 \leq i \leq n$. We denote its minimal period by $T_{i} \quad(p+1 \leq i \leq n)$. We can also assume that $T_{i} \leq \omega$ for $p+1 \leq i \leq q$ and $\omega<T_{i} \leq \omega+1$ for $q+1 \leq i \leq n$. We denote by $k_{i}$ the largest integer such that $k_{i} T_{i} \leq \omega \quad(p+1 \leq i \leq q)$, so that $\left(k_{i}+1\right) T_{i}>\omega \quad(p+1 \leq i \leq q)$. We denote by $x_{i}(\cdot)$ the solution of $(2.14)$ with $x_{i}(0)=z_{i}(p+1 \leq i \leq n)$.

We claim that for each $\omega^{\prime}$ such that

$$
\omega<\omega^{\prime}<\min \left\{\left(k_{p+1}+1\right) T_{p+1}, \ldots,\left(k_{q}+1\right) T_{q}, T_{q+1}, \ldots, T_{n}, \omega+1\right\}:=\tau,
$$


the problem

$$
x^{\prime}=\varphi(x), \quad x(0)=x\left(\omega^{\prime}\right)
$$

has no solution $x(\cdot)$, with $x(t) \in B(0, R)$ for all $t$, other than the equilibria $z_{1}, \ldots, z_{p}$.

Indeed, if $x(\cdot)$ satisfies $(2.15)$ and is contained in $B(0, R)$, then $S=\{x(t)$ : $\left.0 \leq t \leq \omega^{\prime}\right\}$ is a singular orbit of (2.14) contained in $B(0, R)$. If it is not a rest point, then $S=S_{i}$ for some $p+1 \leq i \leq n$ and hence there exists $\alpha_{i} \in \mathbf{R}$ such that

$$
x(t)=x_{i}\left(t+\alpha_{i}\right), \quad 0 \leq t \leq \omega^{\prime} .
$$

In particular,

$$
x_{i}\left(\omega^{\prime}+\alpha_{i}\right)=x_{i}\left(\alpha_{i}\right) .
$$

This is impossible for $q+1 \leq i \leq n$ as then $\omega^{\prime}<T_{i}$ and $T_{i}$ is the smallest period. This is impossible for $p+1 \leq i \leq q$ as in this case $k_{i} T_{i}<\omega^{\prime}<$ $\left(k_{i}+1\right) T_{i}$.

Therefore the claim is proved.

Now, the solutions of $(2.15)$ correspond, by the transformation

$$
y(t)=x\left(\frac{\omega^{\prime}}{\omega} t\right), \quad t \in[0, \omega],
$$

to the solutions of the problem

$$
y^{\prime}(t)=\frac{\omega^{\prime}}{\omega} \varphi(y(t)), \quad y(0)=y(\omega) .
$$

Thus, problem (2.16) has, by construction, no nontrivial (i.e. nonequilibrium) solution on $\operatorname{cl}_{X} \Omega$ and, by assumption, no rest point on $\operatorname{fr}_{X} \Omega$ (as its rest points are the same as those of (2.14) and all its possible solutions in $B(0, R)$ are rest points). Now, as (2.14) has no solution on $\operatorname{fr}_{X} \Omega$, the homotopy invariance of coincidence degree implies that

$$
D_{L}\left(L-N_{\varphi}, \Omega\right)=D_{L}\left(L-\frac{\omega^{\prime}}{\omega} N_{\varphi}, \Omega\right),
$$

for all $\omega \leq \omega^{\prime}<\tau$. Fix some $\omega^{\prime} \in(\omega, \tau)$.

Now, by excision,

$$
D_{L}\left(L-\frac{\omega^{\prime}}{\omega} N_{\varphi}, \Omega\right)=\sum_{\substack{1 \leq j \leq p \\ z_{j} \in \Omega}} D_{L}\left(L-\frac{\omega^{\prime}}{\omega} N_{\varphi}, B\left(z_{j}, \delta\right)\right),
$$

where

$$
\delta=\min \left\{\delta_{1}, \eta / 2\right\}, \quad \eta=\min \left\{d\left(S_{i}, S_{j}\right): 1 \leq i \neq j \leq n\right\} .
$$

Now, the problems

$$
\begin{aligned}
& x^{\prime}(t)=\lambda \frac{\omega^{\prime}}{\omega} \varphi(x(t)), \quad \lambda \in(0,1], \\
& x(0)=x(\omega)
\end{aligned}
$$

have no solution on $\operatorname{fr}_{X} B\left(z_{j}, \delta\right)$.

Indeed, if there exists $\lambda^{*} \in(0,1]$ and $\tilde{x}(\cdot) \in \operatorname{fr}_{X} B\left(z_{j}, \delta\right)$ such that

$$
\tilde{x}^{\prime}(t)=\lambda^{*} \frac{\omega^{\prime}}{\omega} \varphi(\tilde{x}(t)), \quad \tilde{x}(0)=\tilde{x}(\omega),
$$


then

$$
y(t):=\tilde{x}\left(\frac{\omega t}{\lambda^{*} \omega^{\prime}}\right)
$$

will satisfy

$$
y^{\prime}(t)=\varphi(y(t)), \quad y(0)=y\left(\lambda^{*} \omega^{\prime}\right)
$$

and hence $\left\{y(t): t \in\left[0, \lambda^{*} \omega^{\prime}\right]\right\}=S_{i^{*}}$, for some $1 \leq i^{*} \leq n$. Moreover,

$$
\left|y(t)-z_{j}\right| \leq \delta \quad \text { for all } t \in\left[0, \lambda^{*} \omega^{\prime}\right]
$$

so that, by the choice of $\delta, i^{*}=j$ and $y(\cdot)$ is constant and equal to $z_{j}$ for all $t \in\left[0, \lambda^{*} \omega^{\prime}\right]$, a contradiction.

Thus we can argue as in [22, pp. 28-29] and obtain

$$
\begin{aligned}
D_{L}\left(L-\frac{\omega^{\prime}}{\omega} N_{\varphi}, B\left(z_{j}, \delta\right)\right) & =d_{B}\left(-J Q N_{\varphi}, B\left(z_{j}, \delta\right) \cap \mathbf{R}^{m}, 0\right) \\
& =(-1)^{m} d_{B}\left(\varphi, B\left(z_{j}, \delta\right) \cap \mathbf{R}^{m}, 0\right)
\end{aligned}
$$

for $1 \leq j \leq p, z_{j} \in \Omega$. Consequently, from (2.18) we have

$$
\begin{aligned}
D_{L}\left(L-\frac{\omega^{\prime}}{\omega} N_{\varphi}, B\left(z_{j}, \delta\right)\right) & =(-1)^{m} \sum_{\substack{1 \leq j \leq p \\
z_{j} \in \Omega}} d_{B}\left(\varphi, B\left(z_{j}, \delta\right) \cap \mathbf{R}^{m}, 0\right) \\
& =(-1)^{m} d_{B}\left(\varphi, \Omega \cap \mathbf{R}^{m}, 0\right) .
\end{aligned}
$$

The result follows by (2.17), (2.20) and (2.13). The proof is complete.

Theorem 1 is a generalization of Lemma VI.1 in [43], where the case $f_{0}=$ $-\nabla V$, with $V \in C^{1}\left(\mathbf{R}^{m}, \mathbf{R}\right)$ and $\Omega \cap \mathbf{R}^{m}=B(0, r), r>0$, is treated.

We remark that (2.19) holds for any linear orientation preserving isomorphism $J: \mathbf{R}^{m} \rightarrow \mathbf{R}^{m}$ (see [43]), and so (2.7) is independent of the choice of $P, Q, J$, whenever det $J>0$. In the more general case in which $J: \operatorname{Im} Q=$ $\mathbf{R}^{m} \rightarrow \operatorname{ker} L=\mathbf{R}^{m}$ is an arbitrary linear isomorphism, we can write, instead of (2.7),

$$
\left|D_{L}\left(L-M_{0}, \Omega\right)\right|=\left|d_{B}\left(f_{0}, \Omega \cap \mathbf{R}^{m}, 0\right)\right| .
$$

From Theorem 1, using the duality theorems developed in [43, Chapter III] and [31, Chapter III], we can find other relations between the degree of some fixed point operators related to (2.5)-(2.2) and the Brouwer degree of $f_{0}$. To this end, the following maps $\Phi_{i}: Y \rightarrow Y, i=1,2,3$, are defined:

$$
\begin{aligned}
& \Phi_{1}(x)(t):=x(\omega)+\int_{0}^{t} f_{0}(x(s)) d s \\
& \Phi_{2}(x)(t):=x(0)+\int_{0}^{\omega} f_{0}(x(s)) d s+\int_{0}^{t} f_{0}(x(s)) d s \\
& \Phi_{3}(x)(t):=x(0)+(\omega-t) \int_{0}^{\omega} f_{0}(x(s)) d s+\int_{0}^{t} f_{0}(x(s)) d s .
\end{aligned}
$$

All the $\Phi_{i}, i=1,2,3$, are completely continuous and their corresponding fixed points are exactly the solutions of (2.5)-(2.2). Moreover, $\left.\Phi_{3}\right|_{X}: X \rightarrow X$. Let $\Omega \subset Y$ be bounded and open (relatively to $Y$ ). In [43], the following 
equalities are proved, provided that there is no $x \in \mathrm{fr}_{Y} \Omega$, solution of (2.5)(2.2):

$$
\begin{aligned}
\operatorname{deg}\left(I_{Y}-\Phi_{1}, \Omega, 0\right) & =\operatorname{deg}\left(I_{Y}-\Phi_{2}, \Omega, 0\right)=\operatorname{deg}\left(I_{Y}-\Phi_{3}, \Omega, 0\right) \\
& =\operatorname{deg}\left(I_{X}-\Phi_{3} \mid X, \Omega \cap X, 0\right) .
\end{aligned}
$$

Indeed, it is sufficient to apply, respectively, Theorem III.1, Theorem III.4 and Proposition III.5 in [43, Chapter III]. Related results can be found in $[31,28]$.

Now, we have

Corollary 1. Assume that there is no $x \in X \cap \mathrm{fr}_{Y} \Omega$ such that $x^{\prime}=f_{0}(x)$. Then, for $i=1,2,3$,

$$
\operatorname{deg}\left(I_{Y}-\Phi_{i}, \Omega, 0\right)=(-1)^{m} d_{B}\left(f_{0}, \Omega \cap \mathbf{R}^{m}, 0\right) .
$$

Proof. It is sufficient to recall that, by [43, Theorem 111.6, Theorem III.7],

$$
\operatorname{deg}\left(I_{Y}-\Phi_{3}, \Omega, 0\right)=D_{L}\left(L-M_{0}, \Omega \cap X\right)
$$

and then Theorem 1 can be applied.

In [48], the author stated the equality

$$
\operatorname{deg}\left(I_{Y}-\Phi_{1}, \Omega, 0\right)=d_{B}\left(-f_{0}, \Omega \cap \mathbf{R}^{m}, 0\right)
$$

for the case when $\Omega$ is a ball and $f_{0}$ is positively homogeneous of order 1 , assuming that equation (2.5) does not possess nontrivial periodic solutions of any period. Hence, Corollary 1 improves Muhamadiev's theorem in [48, Theorem $5, m=1$ ] (see the next section for a more detailed discussion).

Finally, we give an analogous result for the Poincare map. Suppose that equation (2.5) defines a flow in $\mathbf{R}^{m}$, i.e. assume uniqueness and global existence for the solutions of the Cauchy problems associated to (2.5). For each $z \in \mathbf{R}^{m}$, we denote by $x(\cdot, z)$ the solution of $(2.5)$ with $x(0, z)=z$. Thus, the Poincaré-Andronov operator on $[0, \omega]$ is defined by

$$
U_{0} z:=x(\omega, z) \text {. }
$$

Let $G \subset \mathbf{R}^{m}$ be an open bounded set. Then, the following result holds.

Corollary 2. Assume that $U_{0} z \neq z$ for all $z \in$ fr $G$. Then,

$$
d_{B}\left(I-U_{0}, G, 0\right)=(-1)^{m} d_{B}\left(f_{0}, G, 0\right) .
$$

Proof. We fix $R>0$ such that

$$
R>\sup \{|x(t, z)|: 0 \leq t \leq \omega, z \in \operatorname{cl} G\} .
$$

Then, for $\Omega:=\left\{x \in Y: x(0) \in G,|x|_{\infty}<R\right\}$, we have

$$
\operatorname{deg}\left(I_{Y}-\Phi_{1}, \Omega, 0\right)=d_{B}\left(I-U_{0}, G, 0\right) .
$$

Indeed, (2.25) can be obtained either from [31, Theorem 28.5], observing that $G \subset \mathbf{R}^{m}$ and $\Omega \subset Y$ have a "common core" with respect to the $\omega$-periodic boundary value problem (2.5)-(2.2), or from [43, Theorem III.11, Corollary III.12]. Hence, Corollary 1 can be applied and the thesis follows.

Recall that in [3] and [30] the equality $d_{B}(I-U, G, 0)=d_{B}\left(-f_{0}, G, 0\right)$ is proved under the stronger condition that $x(t, z) \neq z$ for all $t \in(0, \omega]$ and $z \in \operatorname{fr} G$ (that is, assuming that all the points of $\operatorname{fr} G$ are of $\omega$-irreversibility [30]).

Now, we are in position to state some existence results. 
II.2. Continuation theorems. In the sequel, we use the notation introduced at the beginning of the chapter. Recall that

$$
f(t, x ; 0)=f_{0}(x), \quad f(t, x ; 1)=F(t, x) .
$$

First, we give our main result for the solvability of

$$
\begin{gathered}
x^{\prime}=F(t, x), \\
x(0)=x(\omega) .
\end{gathered}
$$

Theorem 2. Let $\Omega \subset X$ be an open-bounded set such that the following conditions are satisfied:

$\left(\mathrm{p}_{1}\right)$ there is no $x(\cdot) \in \mathrm{fr}_{X} \Omega$ such that

$$
x^{\prime}=f(t, x ; \lambda), \quad \lambda \in[0,1) ;
$$

$\left(\mathrm{p}_{2}\right)$

$$
d_{B}\left(f_{0}, \Omega \cap \mathbf{R}^{m}, 0\right) \neq 0 .
$$

Then (2.1)-(2.2) has at least one solution $x(\cdot) \in \mathrm{cl}_{X} \Omega$.

Proof. We use the framework of coincidence degree theory as in Theorem 1. The classical Leray-Schauder continuation theorem [37] could be used instead, by the equivalence stated at the beginning of the $\S$ II. 1 .

Besides the spaces and the operators considered there, we further define $M:=$ $M(x ; \lambda): X \times[0,1] \rightarrow Z:$

$$
M(x ; \lambda)(t):=f(t, x(t) ; \lambda) .
$$

Observe that $M(\cdot ; 0)=M_{0}$.

According to [43, Chapter VI], $M$ is $L$-compact on $\mathrm{cl}_{X} \Omega \times[0,1]$. We remark that $x(\cdot)$ is a solution of $x^{\prime}=f(t, x ; \lambda), \lambda \in[0,1]$, with $x(0)=x(\omega)$, if and only if $x \in \operatorname{dom} L$ is a solution of the coincidence equation $L x=$ $M(x ; \lambda), \lambda \in[0,1]$. In particular, $(2.1)-(2.2)$ is equivalent to $L x=M(x ; 1)$ (according to (2.3)).

Without loss of generality, we suppose that $\left(\mathrm{p}_{1}\right)$ holds for $\lambda \in[0,1]$ in $\left(2.1_{\lambda}\right)$. Otherwise, the result is proved for $x \in \mathrm{fr}_{X} \Omega$. Accordingly, by the definition of $M(\cdot ; \lambda)$ and using $\left(\mathrm{p}_{1}\right)$ we have

$$
L x \neq M(x ; \lambda), \quad \lambda \in[0,1],
$$

for all $x \in \operatorname{dom} L \cap \mathrm{fr}_{X} \Omega$. Thus, we can apply the homotopy property of the coincidence degree and obtain:

$$
D_{L}\left(L-M_{0}, \Omega\right)=D_{L}(L-M(\cdot ; 0), \Omega)=D_{L}(L-M(\cdot ; 1), \Omega) .
$$

Assumption $\left(\mathrm{p}_{1}\right)$ (for $\lambda=0$ ) ensures that Theorem 1 can be applied, so that (2.7), (2.24) and $\left(\mathrm{p}_{2}\right)$ imply

$$
\left|D_{L}(L-M(\cdot ; 1), \Omega)\right|=\left|d_{B}\left(f_{0}, \Omega \cap \mathbf{R}^{m}, 0\right)\right| \neq 0 .
$$

Hence, by the existence property of the coincidence degree, there is $\tilde{x} \in \operatorname{dom} L \cap$ $\Omega$ such that $L \tilde{x}=M(\tilde{x} ; 1)$; thus $\tilde{x}(\cdot)$ is a solution to $(2.1)-(2.2)$, with $\tilde{x}(\cdot) \in$ $\operatorname{dom} L \cap \Omega$. The proof is complete.

An immediate consequence of Theorem 2 which is just based on a (suitable) choice of the set $\Omega \subset X$ is the following: 
Corollary 3. Let $G$ be a bounded open subset of $\mathbf{R}^{m}$. Suppose that the following conditions are satisfied:

$\left(\mathrm{j}_{1}\right) \quad$ ("bound set" condition) for any $x(\cdot)$, solution of $\left(2.1_{\lambda}\right)-(2.2)$ such that $x(t) \in \operatorname{cl} G$ for all $t \in[0, \omega]$, it follows that $x(t) \in G$ for all $t \in[0, \omega]$

$$
d_{B}\left(f_{0}, G, 0\right) \neq 0 .
$$

Then (2.1)-(2.2) has at least one solution $x(\cdot)$ such that $x(t) \in \mathrm{cl} G$, for all $t \in[0, \omega]$.

Proof. It is sufficient to define (in the setting of Theorem 2):

$$
\Omega:=\{x \in X: x(t) \in G \forall t \in[0, \omega]\}
$$

and to check that $\left(\mathrm{p}_{1}\right)$ and $\left(\mathrm{p}_{2}\right)$ are fulfilled. For brevity, we omit the details.

Remark 1. Corollary 3 is a continuation theorem analogous to [39]. Namely, in [39] the bound set condition is required for equation

$$
x^{\prime}=\lambda h(t, x ; \lambda), \quad \lambda \in(0,1),
$$

with $h(t, x ; 1)=F(t, x)$, and, in place of $\left(\mathrm{j}_{2}\right)$, the Brouwer degree of the averaged vector field $\bar{h}_{0}(z):=(1 / \omega) \int_{0}^{\omega} h(s, z ; 0) d s$ is considered.

A comparison between the continuation theorem for (2.25) and Corollary 3 can be made by means of the following examples.

Example 1. Let us consider the plane system

$$
x_{1}^{\prime}=x_{2}, \quad x_{2}^{\prime}=-\mu x_{1}^{+}+\nu x_{1}^{-}+p(t),
$$

with $p \in L^{1}([0, \omega], \mathbf{R}), \mu>0, \nu>0, x^{+}:=\max \{x, 0\}, x^{-}:=\max \{-x, 0\}$, which comes from the study of the equivalent second order scalar equation $x^{\prime \prime}+\mu x^{+}-\nu x^{-}=p(t)$.

It is easy to prove that Corollary 3 can be applied with

$$
f(t, x ; \lambda):=\left(x_{2},-\mu x_{1}^{+}+\nu x_{1}^{-}+\lambda p(t)\right), \quad \lambda \in[0,1],
$$

and $G=B(0, R)$, for $R>0$ sufficiently large, provided that

$$
n\left(\mu^{-1 / 2}+\nu^{-1 / 2}\right) \neq \omega / \pi, \quad \text { for every } n \in \mathbf{N} \text {. }
$$

Indeed, in this case a priori bounds for the $\omega$-periodic solutions are available (see $[13,19])$. On the other hand, if we consider the system

$$
x_{1}^{\prime}=\lambda x_{2}, \quad x_{2}^{\prime}=\lambda\left(-\mu x_{1}^{+}+\nu x_{1}^{-}+p(t)\right), \quad \lambda \in(0,1],
$$

the a priori bounds for the $\omega$-periodic solutions can be found only if

$$
\lambda^{-1} n\left(\mu^{-1 / 2}+\nu^{-1 / 2}\right) \neq \omega / \pi, \quad \text { for every } n \in \mathbf{N} \text { and } \lambda \in(0,1] .
$$

Note that $(2.27)$ holds if and only if $\left(\mu^{-1 / 2}+\nu^{-1 / 2}\right)>\omega / \pi$.

Hence, it is easy to choose $\mu$ and $\nu$ such that (2.26) holds, while (2.27) does not. This elementary example shows that there are situations in which Theorem 2 may be more directly used. In $\S$ III we provide some more substantial applications. 
Example 1 deals with a periodically perturbed autonomous system. In the case of a general nonautonomous equation

$$
x^{\prime}=F(t, x),
$$

a natural choice for the homotopy in applying Theorem 2 is to take

$$
f(t, x ; \lambda)=(1-\lambda) \bar{F}(x)+\lambda F(t, x),
$$

where $\bar{F}$ is the averaged vector field defined by

$$
\bar{F}(x)=\frac{1}{\omega} \int_{0}^{\omega} F(s, x) d s .
$$

Example 2. We consider the problem

$$
\begin{gathered}
x^{\prime}=h(t, x)+p(t), \\
x(0)=x(\omega),
\end{gathered}
$$

where $h:[0, \omega] \times \mathbf{R}^{m} \rightarrow \mathbf{R}^{m}$ is a Carathéodory function positively homogeneous of order $\alpha \neq 1$ in $x$ and $p \in L^{1}\left([0, \omega], \mathbf{R}^{m}\right)$. We define $\bar{h}: \mathbf{R}^{m} \rightarrow \mathbf{R}^{m}$ by

$$
\bar{h}(x)=\frac{1}{\omega} \int_{0}^{\omega} h(s, x) d s
$$

and we assume that $\bar{h}(z) \neq 0$ for $|z|=1$ so that $d_{B}(\bar{h}, B(0, r), 0)$ is defined and constant for each $r>0$. Let us define $H: X \times[0,1] \rightarrow Z$ by

$$
H(x ; \lambda)(t):=(1-\lambda) \bar{h}(x(t))+\lambda h(t, x(t))+\lambda \delta(\alpha) p(t),
$$

where

$$
\delta(\alpha)=\max \left\{0, \frac{1-\alpha}{|1-\alpha|}\right\} .
$$

We first show that there is some $r_{0}>0$ such that, for each $\lambda \in[0,1]$, the equation

$$
L x=H(x ; \lambda)
$$

has no solution $x$ with $|x|_{\infty}=r$, for all $0<r \leq r_{0}$ if $\alpha>1$ and $r \geq r_{0}$ if $\alpha<1$.

If this is not the case, there are sequences $\left(r_{k}\right)$ in $(0,+\infty),\left(x_{k}\right)$ in $X$ and $\left(\lambda_{k}\right)$ in $[0,1]$ such that $\left|x_{k}\right|_{\infty}=r_{k}, r_{k} \leq 1 / k$ if $\alpha>1, r_{k} \geq k$ if $\alpha<1$, and

$$
x_{k}^{\prime}=\left(1-\lambda_{k}\right) \bar{h}\left(x_{k}\right)+\lambda_{k} h\left(t, x_{k}\right)+\lambda_{k} \delta(\alpha) p(t)
$$

( $k \in \mathbf{N}$ ). Letting $u_{k}=x_{k} /\left|x_{k}\right|_{\infty}=x_{k} / r_{k}$, we get

$$
u_{k}^{\prime}=r_{k}^{\alpha-1}\left[\left(1-\lambda_{k}\right) \bar{h}\left(u_{k}\right)+\lambda_{k} h\left(t, u_{k}\right)\right]+\lambda_{k} r_{k}^{-1} \delta(\alpha) p(t)
$$

so that, a.e. on $[0, \omega]$,

$$
\left|u_{k}^{\prime}(t)\right| \leq r_{k}^{\alpha-1} \beta(t)+\gamma(t),
$$

for some $\beta, \gamma \in L^{1}([0, \omega], \mathbf{R})$. Consequently, there are subsequences $\left(\lambda_{j_{k}}\right)$, $\left(u_{j_{k}}\right)$ and $\lambda^{*} \in[0,1], v \in C\left([0, \omega], \mathbf{R}^{m}\right),|v|_{\infty}=1$ such that $\left(u_{j_{k}}\right) \rightarrow v$ uniformly on $[0, \omega]$ and $\left(\lambda_{j_{k}}\right) \rightarrow \lambda^{*}$. From

$$
u_{k}(t)-u_{k}(0)=r_{k}^{\alpha-1} \int_{0}^{t}\left[\left(1-\lambda_{k}\right) \bar{h}\left(u_{k}\right)+\lambda_{k} h\left(s, u_{k}\right)+\lambda_{k} r_{k}^{-\alpha} \delta(\alpha) p(s)\right] d s,
$$


we get

$$
v(t)-v(0)=0, \quad t \in[0, \omega],
$$

so that $v$ is constant and $|v|_{\infty}=1$. From (2.29) we also get

$$
0=\int_{0}^{\omega}\left[\left(1-\lambda_{k}\right) \bar{h}\left(u_{k}\right)+\lambda_{k} h\left(s, u_{k}\right)+\lambda_{k} r_{k}^{-\alpha} \delta(\alpha) p(s)\right] d s
$$

and hence, letting $j_{k} \rightarrow+\infty$,

$$
0=\omega \bar{h}(v)
$$

a contradiction.

Hence,

$$
D_{L}(L-H(\cdot ; 1), B(0, r))=D_{L}(L-H(\cdot ; 0), B(0, r))
$$

and by Theorem 1 and our assumption,

$$
D_{L}(L-H(\cdot ; 0), B(0, r))=(-1)^{m} d_{B}(\bar{h}, B(0, r), 0) \text {. }
$$

Then, if $d_{B}(\bar{h}, B(0,1), 0) \neq 0,(2.28)-(2.2)$ will have at least one solution for each $p \in L^{1}\left([0, \omega], \mathbf{R}^{m}\right)$ if $\alpha<1$ and, when $\alpha>1$, there will be some $\varepsilon_{0}>0$ such that, for $|p|_{1} \leq \varepsilon_{0}$, one has

$$
D_{L}\left(L-H(\cdot ; 1)-p, B\left(0, r_{0}\right)\right)=D_{L}\left(L-H(\cdot ; 1), B\left(0, r_{0}\right)\right) \neq 0
$$

and (2.28)-(2.2) has at least one solution. This last situation is related to earlier work of Halanay [26] and Mawhin [42].

A simple consequence of Corollary 3 is based on the fact that, whenever a priori bounds for the solutions of $\left(2.1_{\lambda}\right)$ can be performed, then the "bound set" condition $\left(\mathrm{j}_{1}\right)$ is satisfied. More precisely, we have

Corollary 4. Assume that there is a compact set $K \subset \mathbf{R}^{m}$ containing all the solutions of $\left(2.1_{\lambda}\right)-(2.2)$ and such that $\left\{z \in \mathbf{R}^{m}: f_{0}(z)=0\right\} \subset K$. Let $G \subset \mathbf{R}^{m}$ be an open bounded set such that $K \subset G$ and suppose that

$$
d_{B}\left(f_{0}, G, 0\right) \neq 0 \text {. }
$$

Then (2.1)-(2.2) has at least one solution with values in $K$.

A result analogous to Corollary 3 can be performed in the case when the phase space is not $\mathbf{R}^{m}$ but a closed convex subset $C$ of $\mathbf{R}^{m}$ with nonempty interior, provided that a flow-invariance condition for the set $C$ is satisfied. More precisely, we have

Theorem 3. Let $G \subset C$ be a bounded set which is open relatively to $C$, where $C \subset \mathbf{R}^{m}$ is a closed convex set with int $C \neq \varnothing$. Assume that the following conditions are satisfied:

(c) for each $u \in \operatorname{fr} C \cap G$ there is $\eta \in N(u, C)$ such that

$$
(f(t, u ; \lambda) \mid \eta) \leq 0 \quad \text { for a.e. } t \in[0, \omega] \text { and } \lambda \in[0,1]
$$

$\left(c_{2}\right)$ for any $x(\cdot), \quad \omega$-periodic solution of

$$
x^{\prime}=f(t, x ; \lambda), \quad \lambda \in[0,1),
$$

such that $x(t) \in \operatorname{cl} G$ for all $t \in[0, \omega]$, it follows that $x(t) \in G$ for all $t \in[0, \omega]$ 
( $\left.c_{3}\right)$ the fixed point index $i_{C}\left(r\left(I+f_{0}\right), G\right)$ is defined and

$$
i_{C}\left(r\left(I+f_{0}\right), G\right) \neq 0
$$

where $r: \mathbf{R}^{m} \rightarrow C$ is the canonical projection. Then (2.1)-(2.2) has at least one solution $x(\cdot)$ such that $x(t) \in \mathrm{cl}_{C} G$, for all $t \in[0, \omega]$.

For the proof of Theorem 3, it is sufficient to use the claims in Theorem 1 and to argue as in [5], where a continuation theorem for the existence of solutions to (2.1)-(2.2) which remain in a convex set is performed. Obviously, Corollary 4 can be modified accordingly.

In $\S I V$ we prove a continuation theorem which is a generalization of Theorem 2 to the case when the phase space is an Euclidean Neighbourhood Retract (ENR); however, the proof of this result is obtained by embedding (2.1)-(2.2) in a functional-analytic framework which is different from [43].

Another consequence of Corollary 3 can be deduced in the case of planar systems $(m=2)$ for which equation $\left(2.1_{\lambda}\right)$ takes the form

$$
x_{1}^{\prime}=x_{2}-\lambda g_{1}\left(x_{1}\right)+\lambda P(t), \quad x_{2}^{\prime}=-g_{2}\left(t, x_{1} ; \lambda\right),
$$

where $g_{1}: \mathbf{R} \rightarrow \mathbf{R}$ and $P:[0, \omega] \rightarrow \mathbf{R}$ are continuous functions and $g_{2}:[0, \omega]$ $\times \mathbf{R} \times[0,1] \rightarrow \mathbf{R}$ satisfies the Carathéodory conditions.

Systems like (2.32) come in a natural way from the study of the parametrized Liénard equation in the scalar case $(x \in \mathbf{R})$

$$
x^{\prime \prime}+\lambda \psi_{1}(x) x^{\prime}+\psi_{2}(t, x ; \lambda)=\lambda p(t),
$$

imposing $g_{1}\left(x_{1}\right):=\int_{0}^{x_{1}} \psi_{1}(s) d s, g_{2}:=\psi_{2}, P(t):=\int_{0}^{t} p(s) d s$ (usually, $\int_{0}^{\omega} p=0$ is also assumed in order to get $P(0)=P(\omega))$. In this particular situation, the following one-sided continuation theorem can be proved.

Corollary 5. Suppose that $g_{2}(t, z ; 0):=g_{2}(z)$ and assume that there are constants $R \geq d>0$ such that

$$
g_{2}(t, z ; \lambda) \cdot z>0, \quad \text { for a.e. } t \in[0, \omega] \text { and all } \lambda \in[0,1),|z| \geq d,
$$

and

$$
\max \left\{x_{1}(t): t \in[0, \omega]\right\} \neq R, \quad \text { for any solution }\left(x_{1}(t), x_{2}(t)\right) \text { of }
$$

$$
\text { (2.30)-(2.2), with } \lambda \in[0,1) \text {. }
$$

Then system (2.30) has at least one $\omega$-periodic solution for $\lambda=1$.

The proof of Corollary 5 can be performed through the construction of an open rectangle $G=(-M, R) \times(-M, M) \subset \mathbf{R}^{2}$ such that condition $\left(\mathrm{j}_{1}\right)$ of Corollary 3 is satisfied with respect to the solutions of $(2.30)-(2.2)$. The choice of the constant $M \geq R$ follows by the estimates developed in [46] and [51]. We omit the rest of the proof referring to these papers for the needed computations. We note that Corollary 5 (or some slight variants of it) is the basic tool for the proof of some recent results concerning the periodic BVP for some Lienard and Duffing equations under one-sided growth restrictions on the restoring term $\psi_{2}$ (see $[15,16])$. Our result also improves [15, Lemma 1].

Remark 2. We point out that the results of this section may be extended to the periodic BVP for $n$th order differential systems:

$$
\begin{gathered}
x^{(n)}+F\left(t, x, x^{\prime}, \ldots, x^{(n-1)}\right)=0, \\
x^{(i)}(0)=x^{(i)}(\omega), \quad i=0,1, \ldots, n-1,
\end{gathered}
$$


with $F:[0, \omega] \times \mathbf{R}^{n m} \rightarrow \mathbf{R}^{m}$, by means of the standard reduction of (2.31)(2.32) to the periodic BVP for a system of $n$ first order equations in $\mathbf{R}^{m}$.

More precisely, we assume that there are $f:[0, \omega] \times \mathbf{R}^{n m} \times[0,1] \rightarrow \mathbf{R}^{m}$, which fulfils the Carathéodory assumptions and $f_{0}: \mathbf{R}^{n m} \rightarrow \mathbf{R}^{m}$, such that

$$
\begin{gathered}
F\left(t, x, x^{\prime}, \ldots, x^{(n-1)}\right)=-f\left(t, x, x^{\prime}, \ldots, x^{(n-1)} ; 1\right), \\
f_{0}\left(x, x^{\prime}, \ldots, x^{(n-1)}\right)=-f\left(t, x, x^{\prime}, \ldots, x^{(n-1)} ; 0\right) .
\end{gathered}
$$

We also define $q_{0}: \mathbf{R}^{m} \rightarrow \mathbf{R}^{m}$, by

$$
q_{0}(z):=f_{0}(z, 0, \ldots, 0), \quad z \in \mathbf{R}^{m} .
$$

Then, we have

Corollary 6. Assume that there is $R>0$ such that

$$
\max \left\{\left|x^{(i)}\right|_{\infty}: i=1, \ldots, n-1\right\}<R,
$$

for all possible solutions $x(\cdot)$ of

$$
x^{(n)}=f\left(t, x, x^{\prime}, \ldots, x^{(n-1)} ; \lambda\right), \quad \lambda \in[0,1),
$$

satisfying the boundary condition (2.32). Suppose that, for $r \geq R$,

$$
d_{B}\left(q_{0}, B(0, r), 0\right) \neq 0 .
$$

Then (2.31)-(2.32) has at least one solution.

The proof follows straightforwardly from Corollary 4, arguing like in [40], and therefore it is omitted.

We recall that in $[14$, p. 677] a similar result has been obtained for a second order scalar equation using a different approach based upon some equivariant degree theory.

As a final result, we give a continuation theorem based on the study of the Poincaré map.

For each $z \in \mathbf{R}^{m}, \lambda \in[0,1]$, we denote by $x(\cdot, z ; \lambda)$ the solution of $x^{\prime}=$ $f(t, x ; \lambda)$ such that $x(0, z ; \lambda)=z$. As usual, to do this, we assume uniqueness and global existence for the solutions of the Cauchy problems associated to $\left(2.1_{\lambda}\right)$. The Poincaré-Andronov operator $U_{\lambda}=U_{\lambda}(z): \mathbf{R}^{m} \rightarrow \mathbf{R}^{m}$ is defined as follows:

$$
U_{\lambda}(z)=x(\omega, z ; \lambda) .
$$

Then, we have

Theorem 4. Let $G \subset \mathbf{R}^{m}$ be open and bounded. Assume that the following conditions are satisfied:

$$
\begin{aligned}
U_{\lambda}(z) \neq z \quad \text { for all } z \in \operatorname{fr} G, \quad \lambda \in[0,1) \\
\\
d_{B}\left(f_{0}, G, 0\right) \neq 0 .
\end{aligned}
$$

Then (2.1)-(2.2) has at least one solution. 
Proof. Without restriction, we can suppose that $\left(\mathrm{m}_{1}\right)$ holds with $\lambda \in[0,1]$. Then, it is sufficient to observe that assumption $\left(\mathrm{m}_{1}\right)$ ensures that the map $\left(I-U_{\lambda}\right)$ is an admissible homotopy, so that, by the homotopy invariance of the Brouwer degree,

$$
d_{B}\left(I-U_{1}, G, 0\right)=d_{B}\left(I-U_{0}, G, 0\right) .
$$

Furthermore, Corollary 2 is applicable, so that

$$
d_{B}\left(I-U_{1}, G, 0\right)=(-1)^{m} d_{B}\left(f_{0}, G, 0\right) .
$$

Hence, there is $z \in \operatorname{cl} G$ such that $U_{1}(z)=z$. The proof is complete.

Extensions to differential-delay equations may be performed as well, combining Theorem 1 with the arguments developed in [41].

\section{Applications}

In this section we deal with the problem of the existence of solutions $x(\cdot)$ to

$$
\begin{aligned}
& x^{\prime}=F(t, x), \\
& x(0)=x(\omega),
\end{aligned}
$$

such that $x(t) \in \operatorname{cl} G$ for all $t \in[0, \omega]$, where $G$ is an open bounded subset of $\mathbf{R}^{m}$.

We state some consequences of Theorem 2 and of its corollaries which illustrate the range of applicability of our main result.

Throughout this section, we assume that the nonlinear field $F$ splits as

$$
F(t, x)=g(x)+e(t, x),
$$

where the function $g: \mathbf{R}^{m} \rightarrow \mathbf{R}^{m}$ is continuous and $e:[0, \omega] \times \mathbf{R}^{m} \rightarrow \mathbf{R}^{m}$ satisfies the Carathéodory assumptions. First, we consider the case of "small perturbations"; then, we study large perturbations of positively homogeneous vector fields.

\section{III.1. Small perturbations.}

Corollary 7. Assume that the following conditions are satisfied:

$\left(\mathbf{k}_{1}\right)$ for any $\omega$-periodic solution $x$ of

$$
x^{\prime}=g(x)
$$

such that $x(t) \in \operatorname{cl} G$ for all $t \in[0, \omega]$, it follows that $x(t) \in G$ for all $t \in$ $[0, \omega]$

$$
d_{B}(g, G, 0) \neq 0 .
$$

Then there is $\varepsilon_{0}>0$ such that, for any forcing term $e(\cdot, \cdot)$ with $|e(\cdot, z)|_{\infty} \leq \varepsilon_{0}$ for all $z \in \operatorname{cl} G$, system (3.1) has at least one $\omega$-periodic solution $x(\cdot)$ such that $x(t) \in \operatorname{cl} G$ for all $t \in[0, \omega]$.

Proof. We apply Corollary 3 with $f_{0}=g$. We imbed (3.1) in the family of parametrized equations

$$
x^{\prime}=f(t, x ; \lambda):=g(x)+\lambda e(t, x), \quad \lambda \in[0,1],
$$

and we claim that there is $\varepsilon_{0}>0$ such that for every function $e(\cdot, z)$ with $|e(\cdot, z)|_{\infty} \leq \varepsilon_{0}$ for all $z \in \operatorname{cl} G$, the set $G$ is a "bound set" for $\left(3.1_{\lambda}\right)$. 
Indeed, assume by contradiction that, for each $n \in \mathbf{N}$, there is a function $e_{n}$ such that $|e(\cdot, z)|_{\infty} \leq 1 / n$ for all $z \in \operatorname{cl} G$ and there is an $\omega$-periodic function $x_{n}(\cdot)$ satisfying

$$
x_{n}^{\prime}=g\left(x_{n}\right)+\lambda_{n} e_{n}\left(t, x_{n}\right), \quad \lambda_{n} \in[0,1],
$$

such that $x_{n}(t) \in \operatorname{cl} G$ for all $t$ and $x_{n}\left(t_{n}\right) \in \operatorname{fr} G$ for some $t_{n} \in[0, \omega]$. By Ascoli-Arzelà's theorem we have that there is an $\omega$-periodic solution $x^{*}(\cdot)$ of (3.4), with $x^{*}(t) \in \operatorname{cl} G \forall t$, such that (up to subsequences) $x_{n} \rightarrow x^{*}$ uniformly on $[0, \omega]$. Moreover, for $t_{n} \rightarrow t^{*}$, we have $x^{*}\left(t^{*}\right) \in \operatorname{fr} G$. Thus, passing to the limit in (3.5), a contradiction with $\left(k_{1}\right)$ is reached and the claim is proved, so that $\left(\mathrm{j}_{1}\right)$ is satisfied for $e(\cdot, \cdot)$ sufficiently small. Thus we can apply Corollary 3 and the proof is complete.

With elementary changes in the proof it can be seen that the result is still true when $e(t, x)=e(t)$ and bounds for $|e|_{1}$ are considered.

Corollary 7 enables us to recover a number of previous results, thanks especially to the rather weak condition $\left(\mathrm{k}_{1}\right)$. For example, $\left(\mathrm{k}_{1}\right)$ is satisfied whenever the flow $\pi^{0}$ induced by (3.4) is dissipative (i.e. there is a compact set $K \subset \mathbf{R}^{m}$ such that for each $x \in \mathbf{R}^{m}$ there is $t_{x} \geq 0$ with $\pi^{0}(t, x) \in K$ for all $\left.t \geq t_{x}\right)$; indeed, if this is the case, then $d_{B}(g, G, 0)=(-1)^{m} \chi\left(\mathbf{R}^{m}\right)=(-1)^{m}$, for every $G \supset K$, where $\chi$ is the Euler-Poincaré characteristic (see [31], [61, Theorem 6.1]). Hence, Corollary 7 guarantees the existence of periodic solutions for small periodic perturbations of autonomous dissipative systems. In this manner, we recover some classical results contained in [11, 28, 56].

Now, we discuss other results for the existence of solutions to (3.1)-(3.2) where some conditions less general than $\left(\mathrm{k}_{1}\right)$ are required.

In the two-dimensional case, J. Cronin [9, 10, 11] and A. C. Lando [32, 33] deal with periodic perturbations of autonomous systems of the form:

$$
x^{\prime}=X(x, y)+\varepsilon E_{1}(t), \quad y^{\prime}=Y(x, y)+\varepsilon E_{2}(t) .
$$

Following Gomory's approach [23], the authors are led to construct a simple closed curve $J$ (containing the origin in its interior) such that the unperturbed system

$$
x^{\prime}=X(x, y), \quad y^{\prime}=Y(x, y)
$$

has no closed orbits intersecting $J$.

Clearly, in this situation $\left(\mathrm{k}_{1}\right)$ is satisfied and condition $\left(\mathrm{k}_{2}\right)$ either is explicitly required (see $[32,33]$ ), or it is an implicit consequence of other hypotheses. For instance, in $[9,10,11]$ it is assumed that "the point at infinity is strongly stable relative to (3.7)". However, in this case it can be proved that $d_{B}((X, Y), B(0, R), 0)=1$, for $R$ sufficiently large. From the above discussion, it follows that Corollary 5 contains all the results proved in [9, Theorem 2], [10, Theorem 6], [11, Theorem 2], [32, Theorem 3], [33, Theorem 3].

On the other hand, we observe that none of the above quoted theorems is suitable for dealing with systems like

$$
x^{\prime}=-y^{3}+\varepsilon E_{1}(t), \quad y^{\prime}=x^{3}+\varepsilon E_{2}(t)
$$

(see [10, p. 159]), while Corollary 7 applies.

We also note that many regularity hypotheses which are required in $[9,10$, $11,32,33]$ are avoided using our approach. 
Another condition (stronger than $\left(\mathrm{k}_{1}\right) \wedge\left(\mathrm{k}_{2}\right)$ ) leading to the existence of $\omega$ periodic solutions of (3.6) was given in [1, Theorem 2], where it is assumed that the origin is an isolated critical point with nonzero index and it is not an isochronous center of period $\omega / k \quad(k \in \mathbf{N})$. In fact, in this case it is sufficient to take $G=B(0, \delta)$, with $\delta>0$ sufficiently small. On the same line, see [3, Theorem 2]. Finally, we mention that, by means of Corollary 7, we can give an easy proof of

Nemitzkii's Conjecture (first settled by A. Halanay [25]). If the autonomous system (3.7) has a limit cycle, then there is least one $\omega$-periodic solution of (3.6), for $\varepsilon$ sufficiently small.

Again, Corollary 7 may be applied, choosing $G$ such that $\operatorname{fr} G$ is "sufficiently close" to the limit cycle.

In the higher dimensional case, Corollary 7 is an improvement of [1, Theorem 1], [3, Theorem 1], [27, Theorem 3.13], where, besides $\left(k_{2}\right)$, various specific conditions are required, such as, e.g. [27],

"The origin is the only critical point of (3.7) in a neighbourhood $G$ of itself, and (3.4) has no periodic solutions of period $\omega^{\prime}, 0<\omega^{\prime} \leq \omega$, passing through points of $\operatorname{fr} G$ ".

Corollary 7 is also a generalization of [62, Theorem 4], [66, Theorem 4.1, $\left(c_{1}\right)$ ], where, instead of $\left(k_{1}\right)$, the existence of a compact isolating neighbourhood $K$ for the flow induced by (3.4) is required.

Indeed, if this is the case, then $G=\operatorname{int} K$ is suitable for the validity of Corollary 7. Again, equation (3.8) provides an example of applicability of our result while [62,66] cannot be used (see also Example 3 below).

We end this subsection with an example of a system which is nondissipative and such that, furthermore, the corresponding autonomous system has the origin as a global center. For related results see [20,53].

Example 3. We deal with the forced nonlinear second order scalar equation:

$$
x^{\prime \prime}+\psi(x)=p(t),
$$

where $\psi: \mathbf{R} \rightarrow \mathbf{R}$ is continuous and $p: \mathbf{R} \rightarrow \mathbf{R}$ is continuous, $\omega$-periodic and such that

$$
\bar{p}:=(1 / \omega) \int_{0}^{\omega} p(s) d s=0 .
$$

As is well-known, equation (3.9) is equivalent to the phase-plane system:

$$
x^{\prime}=y+P(t), \quad y^{\prime}=-\psi(x),
$$

where

$$
P(t):=\int_{0}^{t} p(s) d s .
$$

We assume that the function $\psi$ satisfies:

$$
\begin{aligned}
& \psi(x) \cdot x>0 \text { for }|x| \neq 0, \\
& \lim _{|x| \rightarrow+\infty} \Psi(x)=+\infty, \quad \text { with } \Psi(x):=\int_{0}^{x} \psi(\xi) d \xi .
\end{aligned}
$$

From (3.10) and (3.11), it follows that the origin in $\mathbf{R}^{2}$ is a global center for the autonomous system

$$
\left(x^{\prime}, y^{\prime}\right)=g(x, y):=(y,-\psi(x)),
$$


so that there is no compact isolating neighbourhood $\mathrm{cl} G$ of the origin (with $G$ open).

Moreover, for any open bounded set $G \subset \mathbf{R}^{2}$,

$$
d_{B}(g, G, 0)=1 \text { for } 0 \in G, \quad d_{B}(g, G, 0)=0 \text { for } 0 \notin G .
$$

Hence, Theorem 4.1 in [66] cannot be applied.

On the other hand, in order to use Corollary 7 it is sufficient to find a bound set $G$ for (3.12), i.e. an open bounded set with $0 \in G$ such that there is no $\omega$-periodic solution of (3.12) "tangent" to $\operatorname{fr} G$. To this end, we consider, for any $c>0$, the sublevel set $\Psi_{c}:=\left\{(x, y) \in \mathbf{R}^{2}:(1 / 2) y^{2}+\Psi(x)<c\right\}$. Then, $\operatorname{fr} \Psi_{c}$ is a periodic orbit with minimum period:

$$
T_{c}=\sqrt{2} \int_{d}^{c} \frac{1}{\sqrt{\Psi(c)-\Psi(\xi)}} d \xi, \quad \text { with } d<0<c, \Psi(d)=\Psi(c) .
$$

Hence, it is sufficient to find $c>0$ such that $T_{c} \neq \omega / n$ for all $n \in \mathbf{N}$. Such a choice of $c$ is always possible if the continuous map

$$
\tau=:(0,+\infty) \rightarrow(0,+\infty), \quad c \mapsto T_{c},
$$

is not constant.

Then, the following result follows from Corollary 7:

Proposition 1. For any continuous map $\psi: \mathbf{R} \rightarrow \mathbf{R}$ satisfying (3.10), (3.11) and having a nonconstant associated time-map $\tau$, there is $\varepsilon_{0}>0$ such that equation (3.9) has an $\omega$-periodic solution for every $\omega$-periodic forcing term $p(\cdot)$ with $|p|_{1} \leq \varepsilon_{0}$.

Recall that, if $\psi$ is continuously differentiable and odd, then, by a classical theorem of Urabe [64, $\S 13.3$, Corollary 4$], \tau(\cdot)$ is constant if and only if $\psi$ : $\mathbf{R} \rightarrow \mathbf{R}$ is linear.

III.2. Asymptotically homogeneous systems. In this subsection, we deal with perturbations of autonomous systems with positively homogeneous nonlinearity. More precisely, we consider equations of the form

$$
x^{\prime}=g(x)+e(t, x),
$$

with $g: \mathbf{R}^{m} \rightarrow \mathbf{R}^{m}$ continuous and such that, for some $\alpha>0$,

$$
g(k x)=k^{\alpha} g(x), \text { for all } k>0, x \in \mathbf{R}^{m}
$$

and $e:[0, \omega] \times \mathbf{R}^{m} \rightarrow \mathbf{R}^{m}$ satisfying the Carathéodory conditions and such that

$$
\lim _{|x| \rightarrow+\infty}\left(|e(t, x)| /|x|^{\alpha}\right)=0, \quad \text { uniformly a.e. in } t \in[0, \omega]
$$

Systems of the form (3.13) have been widely studied; see, for instance, [ 31 , $34,36,45,47,48]$. In [31, $\$ 41],[47]$ the more general case in which the function $g$ may depend on $t$ is considered too. However, as we show below, there are situations that can be settled in the framework of Corollary 4 but do not fit in $[31,48]$.

In the first result of this subsection we consider the case of $g$ homogeneous of degree one. 
Corollary 8. Assume $\left(\mathrm{L}_{1}\right),\left(\mathrm{L}_{2}\right)$ with $\alpha=1$. Suppose that the following conditions are satisfied:

$$
x=0 \text { is the only } \omega \text {-periodic solution of }
$$

$$
\begin{aligned}
& x^{\prime}=g(x) \\
& \text { ( } \left.\mathrm{L}_{4}\right) \quad d_{B}\left(g, B\left(0, R_{0}\right), 0\right) \neq 0, \quad \text { for some } R_{0}>0 \text {. }
\end{aligned}
$$

Then there is at least one $\omega$-periodic solution to (3.13).

Note that, from $\left(\mathrm{L}_{3}\right)$, the origin is the unique singular point of $g$, and $\left(\mathrm{L}_{1}\right),\left(\mathrm{L}_{4}\right)$ imply that $d_{B}(g, B(0, R), 0) \neq 0$, for every $R>0$.

Proof. We apply Corollary 4 , with $K=B[0, R]$ for $R>0$ sufficiently large, $G=B\left(0, R_{1}\right), R_{1}>R, f_{0}=g, f(t, x ; \lambda)=g(x)+\lambda e(t, x)$. In order to find $R$ and, as a consequence, to prove the existence of a priori bounds for the solutions of $\left(3.1_{\lambda}\right)$, assume by contradiction that there is a sequence $\left(x_{n}\right)$ of $\omega$-periodic functions, with $\left|x_{n}\right|_{\infty} \rightarrow+\infty$ and such that, for every $n \in \mathbf{N}$,

$$
x_{n}^{\prime}=g\left(\dot{x}_{n}\right)+\lambda_{n} e\left(t, x_{n}\right), \quad \lambda_{n} \in[0,1] .
$$

Now we set, for all $n \in \mathbf{N}$,

$$
y_{n}(\cdot):=x_{n}(\cdot) /\left|x_{n}\right|_{\infty},
$$

so that, dividing (3.15) by $\left|x_{n}\right|_{\infty}$ and using $\left(\mathrm{L}_{1}\right)$ we get

$$
y_{n}^{\prime}=g\left(y_{n}\right)+\left(\lambda_{n} e\left(t, x_{n}\right) /\left|x_{n}\right|_{\infty}\right) .
$$

We observe that we can apply Ascoli-Arzela's theorem; therefore, there exists $y^{*}$, with $\left|y^{*}\right|_{\infty}=1$, such that (up to subsequences) $y_{n} \rightarrow y^{*}$ uniformly on $[0, \omega]$. Thus, taking the limit as $n \rightarrow+\infty$ in (3.16) and using $\left(\mathrm{L}_{2}\right)$ we obtain

$$
\left(y^{*}\right)^{\prime}=g\left(y^{*}\right) \text {. }
$$

Therefore, by $\left(\mathrm{L}_{3}\right), y^{*}=0$, which is a contradiction.

Thus, we have proved that there is $R>0$ such that, for every $\omega$-periodic solution $x(\cdot)$ of $(3.14), \quad x(t) \in B[0, R]$ for all $t \in[0, \omega]$. Therefore, using $\left(\mathrm{L}_{4}\right)$ we see that Corollary 4 is applicable and we get the existence of an $\omega$ periodic solution of (3.13) such that $x(t) \in B[0, R]$ for all $t \in[0, \omega]$. The proof is complete.

Remark 3. Corollary 8 is a generalization of the results (in the case $\alpha=1$ ) in [48], where, besides $\left(L_{1}\right),\left(L_{2}\right)$ and $\left(L_{4}\right)$, the fact that there are no cycles or nontrivial equilibrium states for the autonomous system $x^{\prime}=g(x)$ is assumed. In other words, $\left(\mathrm{L}_{3}\right)$ must hold for periodic solutions of any period. Hence, the range of applicability of our corollary is wider than Muhamadiev's one. For instance, Muhamadiev's theorem does not apply to Example 1 in $\S I I$; indeed, in that situation, for $\mu, \nu>0$, the origin is a global center for the autonomous system

$$
x_{1}^{\prime}=x_{2}, \quad x_{2}^{\prime}=-\mu x_{1}^{+}+\nu x_{1}^{-},
$$

while our result applies, provided that $(\mu, \nu)$ does not belong to the DancerFučik spectrum [19].

We also point out that, apparently, Corollary 8 (or, more precisely, the evaluation of the Leray-Schauder degree of the Nemitzkii operator induced by (3.14) 
in terms of the Brouwer degree of $g$ ) cannot be obtained by means of the techniques developed in [31]. To this regard, see the problem raised in [31, p. 256].

Now, we state the analogue of Corollary 8 for $n$th order systems of the form

$$
x^{(n)}+F\left(x, x^{\prime}, \ldots, x^{(n-1)}\right)=e\left(t, x, x^{\prime}, \ldots, x^{(n-1)}\right) .
$$

Corollary 9. Assume that the following conditions are satisfied:

$\left(\mathrm{f}_{1}\right) F\left(k x, k x^{\prime}, \ldots, k x^{(n-1)}\right)=k F\left(x, x^{\prime}, \ldots, x^{(n-1)}\right)$, for all $k>0$ and $\left(x, x^{\prime}, \ldots, x^{(n-1)}\right) \in \mathbf{R}^{n m}$;

(f $_{2} \lim _{|x|+\left|x^{\prime}\right|+\cdots+\left|x^{(n-1)}\right| \rightarrow+\infty}\left|e\left(t, x, x^{\prime}, \ldots, x^{(n-1)}\right)\right| /\left(|x|+\left|x^{\prime}\right|+\cdots+\left|x^{(n-1)}\right|\right)=0$, uniformly a.e. in $t \in[0, \omega]$;

$\left(\mathrm{f}_{3}\right) x(t)=0$, for all $t \in[0, \omega]$, is the only $\omega$-periodic solution of

$$
x^{(n)}+F\left(x, x^{\prime}, \ldots, x^{(n-1)}\right)=0 .
$$

$\left(\mathrm{f}_{4}\right) d_{B}(q, B(0, r), 0) \neq 0$, for $r>0$, where $q(x):=F(x, 0, \ldots, 0)$.

Then, (3.17) has at least one $\omega$-periodic solution.

As for the proof, it is sufficient to repeat the argument in the proof of Corollary 9 and to apply Corollary 6.

Remark 4. The particular case when $q(x)=F(x, 0, \ldots, 0)=\nabla V(x)$, with $V: \mathbf{R}^{m} \rightarrow \mathbf{R}$ a positively homogeneous potential of degree 2 has been considered by many authors (see, e.g., [31, §12.4], [36]). Corollary 9 improves the result in [36], where system

$$
x^{\prime \prime}+\nabla V(x)=p(t)
$$

is studied. Indeed, besides assumptions analogous to $\left(\mathrm{f}_{1}\right),\left(\mathrm{f}_{2}\right),\left(\mathrm{f}_{3}\right)$, it is assumed in [36] that $V(x)>0$ for $x \neq 0$ so that $\left(\mathrm{f}_{4}\right)$ holds as well (see [31, Theorem 12.6]). This remark shows that Corollary 9 contains the classical theorems in [13, 19] on jumping nonlinearities, where asymptotically homogeneous autonomous equations are considered, and some of the results in [17, 18]. An easier proof of the theorem in [36] has been recently obtained in [45].

As a second consequence of Corollary 4, we perform a result for asymptotically positively homogeneous systems of order $\alpha$, with $\alpha \neq 1$.

Corollary 10. Assume $\left(\mathrm{L}_{1}\right),\left(\mathrm{L}_{2}\right),\left(\mathrm{L}_{4}\right)$ and suppose, respectively, either

$$
x=0 \text { is the only bounded solution of } x^{\prime}=g(x) \quad(\text { if } \alpha>1) \text {; }
$$

or

$$
g(x) \neq 0 \quad \text { for } x \neq 0 \quad(\text { if } \alpha<1) .
$$

Then, (3.13) has at least one $\omega$-periodic solution.

The proof can be obtained by repeating essentially the proof of Corollary 8 , or following Muhamadiev's argument [48].

By means of [48] and Corollary 4 it is easy to extend the result to systems of the form

$$
x^{\prime}=g(t, x)+e(t, x)
$$


using a homotopy between (3.18) and either the "freezed" system $x^{\prime}=g(0, x)$ for $\alpha>1$, or the "averaged" system $x^{\prime}=\bar{g}(x)$ for $\alpha<1$.

We do not give any new contribution for (3.18), except for the abstract theorem we use; hence, we do not state such results in detail.

Finally, we mention that by means of the (continuation) Theorem 2 it is possible to obtain a result on the so-called "regular guiding functions" (see [4], $[31, \S 14])$. In this way, we can easily recover $[8,12,23]$, where a perturbation of a polynomial in $\mathbf{R}^{2}$ is studied.

\section{AN EXTENSION TO FLOW-INVARIANT ENRS}

In a recent paper [6], a variant of Mawhin's continuation theorem has been obtained for differential systems inducing a flow on some closed ENRs. In what follows we give a similar extension of Theorem 2, provided that a Kupka-Smale approximation property holds.

Let $C \subset \mathbf{R}^{m}$ be a closed ENR. In this section, our goal is to prove the existence of a solution $x(\cdot)$ to

$$
\begin{aligned}
& x^{\prime}=F(t, x), \\
& x(0)=x(\omega),
\end{aligned}
$$

such that, for all $t \in[0, \omega], x(t)$ belongs to a certain subset of $C$.

The particular case when $C=\mathbf{R}^{m}$ has been treated in the previous sections.

As before, we assume that

$$
F(t, x):=f(t, x ; 1),
$$

where

$$
f=f(t, x ; \lambda):[0, \omega] \times C \times[0,1] \rightarrow \mathbf{R}^{m}
$$

is a continuous function which is locally lipschitzian in $x$, uniformly in $t, \lambda$. Once for all, we point out that such assumption is not strictly necessary in our proofs, but it provides the uniqueness of the solutions to all the Cauchy problems which we will consider henceforth. Moreover, we assume that for $\lambda=0$ the map $f$ is autonomous, i.e. there exists a function $f_{0}: C \rightarrow \mathbf{R}^{m}$ such that

$$
f_{0}(x)=f(t, x ; 0),
$$

for all $t \in[0, \omega], x \in C$.

In this more general situation, we need a "flow-invariance" hypothesis ensuring that system

$$
x^{\prime}=f(t, x ; \lambda)
$$

induces a local process in $C$, for all $\lambda \in[0,1]$. More precisely, we want that, for each $\left(t_{0}, x_{0}\right) \in[0, \omega) \times C$ and for all $\lambda \in[0,1]$, the Cauchy problem

$$
x^{\prime}=f(t, x ; \lambda), \quad x\left(t_{0}\right)=x_{0},
$$

has a solution $x(\cdot): \operatorname{dom} x(\cdot) \rightarrow C$ defined on a right maximal neighbourhood of $t_{0}$. if

Nagumo's theorem (see $[49,67])$ ensures that this fact holds true if and only

$$
f(t, z ; \lambda) \in T(z ; C) \quad \text { for all } t \in[0, \omega], \quad z \in \text { fr } C, \lambda \in[0,1],
$$


where $T(z ; C)$ is the (Bouligand) tangent cone to $C$ at $z$. In other words, condition $\left(i_{1}\right)$ means that the function $f$ is "subtangential" to $C$ at $z \in \operatorname{fr} C$.

Remark 5. Assumption $\left(i_{1}\right)$ is obviously satisfied in the case when $C=\mathbf{R}^{m}$. If set $C$ is a regular manifold and $f(t, \cdot ; \lambda)$ is a tangent vector field, then $f(t, z ; \lambda) \in T(z ; C) \cap-T(z ; C)$, so that $\left(\mathrm{i}_{1}\right)$ holds (see [21]). If $C$ is a closed convex set with nonempty interior (like in [5]) then $\left(i_{1}\right)$ can be written as

$$
(f(t, z ; \lambda) \mid \eta) \leq 0 \text { for all } t \in[0, \omega], z \in \text { fr } C, \lambda \in[0,1] \text { and } \eta \in N(z) \text {. }
$$

Now, we introduce the crucial "approximation"

Property (A). If

$$
f_{0}(z) \in T(z ; C) \text { for all } z \in \text { fr } C,
$$

then there exists a sequence of locally lipschitzian functions $\left(\varphi_{k}\right), \varphi_{k}: C \rightarrow \mathbf{R}^{m}$ such that:

(a') $\varphi_{k}(z) \in T(z ; C)$ for all $z \in$ fr $C, k \in \mathbf{N}$;

$\left(\mathrm{b}^{\prime}\right) \quad\left(\varphi_{k}\right) \rightarrow f_{0}$ uniformly on compact sets;

(c') for every compact subset $K$ of $C$ and for all $k \in \mathbf{N}$, system

$$
x^{\prime}=\varphi_{k}(x)
$$

has finitely many singular orbits (i.e., rest points and closed orbits) with minimal period in $[0, \omega+1]$ which are contained in $K$.

We stress the fact that if the set $C$ is a manifold (with or without boundary) and $f_{0}$ is a tangent vector field to $C$, then property $(\mathrm{A})$ is satisfied. Indeed, this is a consequence of the Kupka-Smale theorem. In particular, property (A) is satisfied in the case when $C=\mathbf{R}^{m}$ (as in $\S$ II above). If the set $C$ is a closed convex set with nonempty interior (as in [5]), then it is easy to prove that property $(A)$ is satisfied. Indeed, if (4.5) holds one can show, by a standard perturbation argument, that there are sequences $\left(\psi_{k}\right)$ and $\left(\delta_{k}\right) \downarrow 0$ such that $\psi_{k} \rightarrow f_{0}$ uniformly on compact sets and $\left(\psi_{k}(z) \mid \eta\right) \leq-\delta_{k}<0$ for all $z \in \operatorname{fr} C, \eta \in N(z)$. Now, by the Kupka-Smale theorem, we have that, for each $k \in \mathbf{N}$, there is a sequence $\left(\varphi_{k, n}\right)_{n=0}^{\infty}$ satisfying $\left(\mathbf{a}^{\prime}\right),\left(\mathbf{b}^{\prime}\right)$ and $\left(\mathbf{c}^{\prime}\right)$. Finally, a diagonal argument leads to the conclusion.

Before stating our main result, we recall the definition of the "index of rest points".

Assume $\left(i_{1}\right)$ and consider system

$$
x^{\prime}=f_{0}(x) \text {. }
$$

The local lipschitzian continuity of the function $f_{0}$ ensures that (4.8) induces a local semidynamical system $\pi^{0}$ with phase space $C$. We recall that if $\left(i_{1}\right)$ holds then, taking $\lambda=0$ in $\left(i_{1}\right)$, it is immediately seen that the set $C$ is positively invariant for (4.8). Let $G \subset C$ be a bounded set, open relatively to C. If

$$
f_{0}(x) \neq 0 \text { for all } x \in \mathrm{fr}_{C} G,
$$

then there exists $\varepsilon_{0}>0$ such that the " $\varepsilon$-Poincare map" $\pi_{\varepsilon}^{0}: x \mapsto \pi^{0}(\varepsilon, x)$ is fixed point free on the set $\operatorname{fr}_{C} G$, for all $0<\varepsilon \leq \varepsilon_{0}$. Hence, the fixed point 
index $i_{C}\left(\pi_{\varepsilon}^{0}, G\right)$ is defined and it is constant with respect to $\varepsilon \in\left(0, \varepsilon_{0}\right]$ (see [61]). Thus, the integer

$$
I\left(\pi^{0}, G\right):=\lim _{\varepsilon \rightarrow 0^{+}} i_{C}\left(\pi_{\varepsilon}^{0}, G\right)
$$

is well defined, and it is called the "index of rest points".

Remark 6. We recall some facts about the computation of the index of rest points. If $G=C(C$ compact $)$, then $I\left(\pi^{0}, G\right)=\chi(C)$, where $\chi$ denotes the Euler-Poincaré characteristic (see [21,61]). If cl $G \subset$ int $C$, then $I\left(\pi^{0}, G\right)=$ $(-1)^{m} d_{B}\left(f_{0}, G, 0\right)$ (see $\left.[31,61]\right)$. If $C$ is a closed convex set with nonempty interior, then $I\left(\pi^{0}, G\right)=i_{C}\left(r\left(I+f_{0}\right), G\right), r: \mathbf{R}^{m} \rightarrow C$ being the canonical projection (see [5]). If $C$ is a manifold (satisfying suitable assumptions) and $f_{0}$ is a vector field tangent to $C$, then $I\left(\pi^{0}, G\right)=\chi\left(-f_{0}\right)$, where $\chi$ is the "characteristic of the vector field" $f_{0}$ introduced in [21].

In what follows, we denote by $\Gamma$ the complete metric space of the continuous functions $x(\cdot):[0, \omega] \rightarrow C$ endowed with the distance $d^{*}, d^{*}\left(x_{1}, x_{2}\right):=$ $\left|x_{1}-x_{2}\right|_{\infty}$. We recall the following crucial result (see [29]): the space $\left(\Gamma, d^{*}\right)$ is a metric ANR if and only if the set $C$ is an ANR. This theorem will enable us to work with the fixed point index of compact operators defined in the function space $\Gamma$. Notice that in what follows points of $C$ will be identified with constant functions. Now, we are in position to state our main result.

Theorem 5. Assume $\left(i_{1}\right)$ and (A). Let $\Omega \subset \Gamma$ be an open bounded set such that the following conditions are satisfied:

$$
\text { there is no } x(\cdot) \in \mathrm{fr}_{\Gamma} \Omega \text {, with } x(0)=x(\omega) \text {, such that }
$$

$$
\begin{gathered}
x^{\prime}=f(t, x ; \lambda), \quad \lambda \in[0,1) ; \\
I\left(\pi^{0}, \Omega \cap G\right) \neq 0 .
\end{gathered}
$$

Then (4.1)-(4.2) has at least one solution $x(\cdot) \in \mathrm{cl}_{\Gamma} \Omega$.

Proof. We begin by observing that, as $\Omega$ is bounded, there is a constant $R>0$ such that

$$
|x|_{\infty}<R, \quad \text { for every } x \in \mathrm{cl}_{\Gamma} \Omega .
$$

Now, consider a sequence of locally lipschitzian functions $\left(\varphi_{k}\right), \varphi_{k}: C \rightarrow$ $\mathbf{R}^{m}$, with $\varphi_{k} \rightarrow f_{0}$, uniformly on $C \cap B[0, R]$, and satisfying $\left(\mathbf{a}^{\prime}\right)$ and $\left(\mathbf{c}^{\prime}\right)$, according to property (A).

As a first step, we claim that, without loss of generality, we can suppose that

$\left(\mathrm{d}^{\prime}\right)$ for each $k \in \mathbf{N}$, the problem

$$
x^{\prime}=\varphi_{k}(x), \quad x(0)=x(\omega)
$$

has no nontrivial solution in $C \cap B(0, R)$.

Indeed, consider the singular orbits (i.e. rest points and closed orbits) with minimal period in $[0, \omega+1]$ of the system $x^{\prime}=\varphi_{k}(x)$. By $\left(\mathrm{c}^{\prime}\right)$, there exist finitely many such orbits $S_{1}, \ldots, S_{n_{k}}$, which are contained in $C \cap B(0, R)$. Let $z_{i}\left(1 \leq i \leq p_{k}, p_{k} \geq 0\right.$ an integer) be the rest points among the $S_{i}$. Arguing as in the proof of Theorem 1 (from step (2.14) to step (2.17)), we can find, for each $k \in \mathbf{N}$, a constant $\tau_{k}>\omega$ such that, for each $\omega<\omega^{\prime}<\tau_{k}$, the problem

$$
x^{\prime}=\varphi_{k}(x), \quad x(0)=x\left(\omega^{\prime}\right)
$$


has no solution $x(\cdot)$, with $x(t) \in C \cap B(0, R)$ for all $t$, other than the equilibria $z_{1}, \ldots, z_{p_{k}}$.

Hence, if we choose, for each $k \in \mathbf{N}$,

$$
\omega<\omega_{k}^{\prime}<\min \left\{\tau_{k}, \omega+\frac{1}{k}\right\}
$$

and define

$$
\varphi_{k}^{*}(z):=\left(\omega_{k}^{\prime} / \omega\right) \varphi_{k}(z), \quad \text { for each } z \in C,
$$

we get a sequence of locally lipschitzian functions $\left(\varphi_{k}^{*}\right), \varphi_{k}^{*}: C \rightarrow \mathbf{R}^{m}$, with $\varphi_{k}^{*} \rightarrow f_{0}$, uniformly on $C \cap B[0, R]$, which satisfies $\left(\mathbf{a}^{\prime}\right)$ (by the cone property of $T(z ; C))$ and such that the problem

$$
x^{\prime}=\varphi_{k}^{*}(x), \quad x(0)=x(\omega),
$$

has no nontrivial solution in $C \cap B(0, R)$. The claim is thus proved.

Then, in the sequel, we can assume $\left(\mathrm{d}^{\prime}\right)$.

As a next step, we proceed along the lines of the proof of Theorem 1 in [6].

We suppose that $\left(i_{2}\right)$ holds with $\lambda \in[0,1]$ in $\left(4.1_{\lambda}\right)$ (otherwise, the result is proved for $x \in \operatorname{fr}_{\Gamma} \Omega$ ).

Let us consider the Cauchy problem

$$
\begin{gathered}
y^{\prime}=f(t, y ; \lambda), \\
y(\sigma)=z .
\end{gathered}
$$

Without loss of generality, we can assume $f$ bounded, possibly replacing it by a modified function like $f(t, x ; \lambda) \rho(|x|)$, as in the proof of Theorem 1 in [6]. Since, in this situation, uniqueness and global existence for (4.10)-(4.11) are guaranteed, then if we denote by $u(\sigma, z, \cdot ; \lambda)$ the solution of (4.10)-(4.11) a one-parameter family of processes is defined.

Besides, we introduce a one-parameter family of compact operators defined on $\Gamma \times[0,1]$ as follows:

$$
M(x ; \lambda):=u(0, x(\omega), \cdot ; \lambda), \quad \lambda \in[0,1] .
$$

By $\left(\mathrm{i}_{1}\right), \quad M: \Gamma \times[0,1] \rightarrow \Gamma$; furthermore, Ascoli-Arzela's theorem ensures that $M$ is compact on $\operatorname{cl}_{\Gamma} \Omega \times[0,1]$. By the definition of $M$, it is easily seen that $x$ is a fixed point of $M(\cdot ; \lambda)$ if and only if $x(\cdot)$ is a solution of (4.10) such that $x(0)=x(\omega)$. Accordingly, our aim is to prove the existence of a fixed point of the operator $M(\cdot ; 1)$. This fact, together with $(4.3)$, implies the thesis.

By assumption $\left(\mathrm{i}_{2}\right)$, we have that $M(x ; \lambda) \neq x$ for all $x \in \operatorname{fr}_{\Gamma} \Omega$ and $\lambda \in[0,1]$, so that $M$ is an admissible homotopy and

$$
i_{\Gamma}(M(\cdot ; 1), \Omega)=i_{\Gamma}(M(\cdot ; 0), \Omega) .
$$

Observe that the existence of a fixed point of the operator $M(\cdot ; 0)$ is equivalent to the existence of an $\omega$-periodic solution of the autonomous system (4.8).

Now, by property (A) and the preceding claim, there is a sequence $\left(\varphi_{k}\right)$ satisfying $\left(\mathrm{a}^{\prime}\right),\left(\mathrm{b}^{\prime}\right),\left(\mathrm{c}^{\prime}\right)$ and $\left(\mathrm{d}^{\prime}\right)$, relatively to $C \cap B[0, R]$. Let us denote, for every $k \in \mathbf{N}$, by $\pi^{k, \mu}$ the dynamical system induced by

$$
y^{\prime}=\mu f_{0}(y)+(1-\mu) \varphi_{k}(y),
$$

with $\mu \in[0,1]$. 
Thus, for each $k \in \mathbf{N}$, a one-parameter family of dynamical systems is defined. Assumptions $\left(i_{1}\right)$ and (A) imply that the set $C$ is flow-invariant for the dynamical systems $\pi^{k, \mu}$ as well.

Indeed, this follows from the convexity and the cone property of some tangent cones which can be equivalently used in place of $T(z ; C)$ (cf. Lemma 1 in [6] for the detailed proof of an analogous result).

On the other hand, assumption $\left(i_{2}\right)$ implies that $f_{0}(z) \neq 0$ for all $z \in$ $\operatorname{fr}_{\Gamma} \Omega \cap C$; hence, by the compactness of $\operatorname{fr}_{\Gamma} \Omega \cap C$, we obtain that there is $\varepsilon_{0} \in(0, \omega)$ such that

$$
\pi^{k, 0}(\varepsilon, z)=\pi^{0}(\varepsilon, z)=\pi_{\varepsilon}^{0}(z) \neq z,
$$

for all $z \in \mathrm{fr}_{\Gamma} \Omega \cap C, \varepsilon \in\left(0, \varepsilon_{0}\right]$.

Now, as before, we introduce, for every $k \in \mathbf{N}$, an operator $N^{k}$ defined on $\Gamma \times[0,1]$ by

$$
N^{k}(x ; \mu):=\pi^{k, \mu}(\cdot, x(\omega)) ;
$$

observe that $N^{k}: \Gamma \times[0,1] \rightarrow \Gamma$ and that it is compact on $\operatorname{cl}_{\Gamma} \Omega \times[0,1]$. Moreover,

$$
N^{k}(\cdot ; 1)=M(\cdot ; 0),
$$

for all $k \in \mathbf{N}$.

We claim that there is $k_{0}$ such that, for all $k \geq k_{0}$ and for all $\mu \in[0,1]$, $N^{k}(\cdot ; \mu)$ is an admissible homotopy. This fact will imply, in particular, that if we denote by $\pi^{k}:=\pi^{1, k}$ the flow induced by $x^{\prime}=\varphi_{k}(x)$, then

$$
\pi^{k}\left(\varepsilon_{0}, z\right) \neq z, \quad \text { for all } z \in C \cap \mathrm{fr}_{\Gamma} \Omega, \quad k \geq k_{0} .
$$

Moreover, a classical compactness argument implies that, for any $k \geq k_{0}$, there is $\delta_{1}=\delta_{1}(k)>0$ such that

$$
\pi^{k}\left(\varepsilon_{0}, y\right) \neq y, \quad \text { for all } y \in B\left(\mathrm{fr}_{\Gamma} \Omega \cap C, \delta_{1}\right) .
$$

Indeed, it is sufficient to observe that the sequence of operators $\left(N^{k}\right)$ converges to $M(\cdot ; 0)$ uniformly on $\mathrm{cl}_{\Gamma} \Omega \times[0,1]$ and that

$$
\inf \left\{d^{*}(x, M(x ; 0)): x \in \mathrm{fr}_{\Gamma} \Omega\right\}>0
$$

(recall that $M(\cdot ; 0)$ is compact on $\operatorname{fr}_{\Gamma} \Omega$ and $\operatorname{fr}_{\Gamma} \Omega$ is closed). Hence, the claim is proved and we can write

$$
i_{\Gamma}\left(N^{k}(\cdot ; 1), \Omega\right)=i_{\Gamma}\left(N^{k}(\cdot ; 0), \Omega\right),
$$

for every $k \geq k_{0}$ and, in particular,

$$
i_{C}\left(\pi_{\varepsilon_{0}}^{k}, \Omega \cap C\right)=i_{C}\left(\pi_{\varepsilon_{0}}^{0}, \Omega \cap C\right) .
$$

Let us fix $k^{*} \geq k_{0}$. For brevity, we set $\varphi:=\varphi_{k^{*}}, N:=N^{k^{*}}, \pi^{\varphi}:=\pi^{k^{*}}, \delta_{1}:=$ $\delta_{1}\left(k^{*}\right)$. Let $S_{1}, \ldots, S_{n} \subset C \cap B(0, R)$ be the singular orbits (i.e. rest points and closed orbits) with minimal period in $[0, \omega+1]$ of the dynamical system $\pi^{\varphi}$ induced by

$$
x^{\prime}=\varphi(x),
$$

which are contained in $C \cap B(0, R)$. 
Arrange the indexes so that $z_{i}$, for $1 \leq i \leq p$, are the rest points of $\pi^{\varphi}$ in $C \cap B(0, R)$, that is $z_{i}=S_{i}$, for $i=1, \ldots, p$.

By $\left(\mathrm{d}^{\prime}\right)$, we know that $x(\cdot) \in \mathrm{cl}_{\Gamma} \Omega$ is a fixed point of the operator $N(\cdot ; 0)$ if and only if $x(t)=z_{i}$, for all $t \in[0, \omega]$, with $z_{i} \in \Omega \cap C$. Hence, by excision,

$$
i_{\Gamma}(N(\cdot ; 0), \Omega)=\sum_{\substack{1 \leq j \leq p \\ z_{j} \in \Omega}} i_{\Gamma}\left(N(\cdot ; 0), B\left(z_{j}, \delta\right)\right)
$$

where

$$
\delta=\min \left\{\delta_{1}, \eta / 2\right\}, \quad \eta=\min \left\{d\left(S_{i}, S_{j}\right): 1 \leq i \neq j \leq n\right\} .
$$

Now, we introduce a third homotopy by whom, roughly speaking, we "move along the orbits" of the dynamical system $\pi^{\varphi}$.

We define an operator $H$ on $\Gamma \times[0,1]$ as follows:

$$
H(x ; \beta):=\pi^{\varphi}\left(x(\omega),(1-\beta) \varepsilon_{0}+\beta \cdot\right) .
$$

As before, $H: \Gamma \times[0,1] \rightarrow \Gamma$ and it is compact on $\operatorname{cl}_{\Gamma} \Omega \times[0,1]$. Moreover,

$$
N(\cdot ; 0)=H(\cdot ; 1) \text {. }
$$

We observe that $x$ is a fixed point of $H(\cdot ; \beta)$ if and only if $x(t) \equiv$ $y((1-\beta) \varepsilon+\beta t)$, with $y(\cdot)$ a $\gamma_{0}$-periodic solution of

$$
y^{\prime}=\varphi(y), \quad y(0)=x(\omega)
$$

and $\gamma_{0}:=(1-\beta) \varepsilon+\beta \omega$.

By the same argument used in the proof of Theorem 1 (from step (2.18) to step (2.19)), we have that $H(\cdot ; \beta)$ has no fixed points on $\operatorname{fr}_{\Gamma} B\left(z_{j}, \delta\right)$, for each $j=1, \ldots, p \quad\left(z_{j} \in \Omega\right)$ and each $\beta \in[0,1]$, so that

$$
i_{\Gamma}\left(H(\cdot ; 1), B\left(z_{j}, \delta\right)\right)=i_{\Gamma}\left(H(\cdot ; 0), B\left(z_{j}, \delta\right)\right) .
$$

Since the only fixed points of $H(\cdot ; 0)$ in $\mathrm{cl}_{\Gamma} \Omega$ are the $z_{j}, j=1, \ldots, p$ $\left(z_{j} \in \Omega\right)$, by excision it follows that

$$
i_{\Gamma}(H(\cdot ; 0), \Omega)=\sum_{\substack{1 \leq j \leq p \\ z_{j} \in \Omega}} i_{\Gamma}\left(H(\cdot ; 0), B\left(z_{j}, \delta\right)\right),
$$

Therefore, from $(4.18),(4.19),(4.20)$ and (4.21) we obtain that

$$
i_{\Gamma}(N(\cdot ; 0), \Omega)=i_{\Gamma}(H(\cdot ; 0), \Omega) .
$$

Since $H(\cdot ; 0): \Gamma \rightarrow C$, then by the contraction property of the fixed point index (see [50]) we have

$$
i_{\Gamma}(H(\cdot ; 0), \Omega)=i_{C}(H(\cdot ; 0), \Omega \cap C) .
$$

Furthermore, using the fact that

$$
H(x ; 0)=\pi^{\varphi}\left(\varepsilon_{0}, x(\omega)\right)=\pi_{\varepsilon_{0}}^{\varphi}(x(\omega)),
$$

we get

$$
i_{C}(H(\cdot ; 0), \Omega \cap C)=i_{C}\left(\pi_{\varepsilon_{0}}^{\varphi}, \Omega \cap C\right) .
$$

Finally, by the choice of $k^{*}$, we can use $(4.16)$, so that 


$$
i_{C}\left(\pi_{\varepsilon_{0}}^{\varphi}, \Omega \cap C\right)=i_{C}\left(\pi_{\varepsilon_{0}}^{0}, \Omega \cap C\right)=i_{C}\left(\pi_{\varepsilon}^{0}, \Omega \cap C\right),
$$

for $\varepsilon \in\left(0, \varepsilon_{0}\right]$.

In conclusion, we have proved that $i_{\Gamma}(M(\cdot ; 1), \Omega)=i_{C}\left(\pi_{\varepsilon}^{0}, \Omega \cap C\right)$ is constant with respect to $\varepsilon$, for $\varepsilon>0$ small enough.

Then,

$$
i_{\Gamma}(M(\cdot ; 1), \Omega)=\lim _{\varepsilon \rightarrow 0^{+}} i_{C}\left(\pi_{\varepsilon}^{0}, \Omega \cap C\right)=I\left(\pi^{0}, \Omega \cap C\right) .
$$

Assumption $\left(\mathrm{i}_{3}\right)$ provides the existence of a fixed point $x \in \Omega$ of $M(\cdot ; 1)$. The proof is complete.

Remark 7. We point out that a generalization of Corollaries 3 and 4 to the case of flow-invariant ENRs can be performed arguing as in $\S$ II. Besides an analogous "bound set" or "a priori bounds" condition (respectively), it is sufficient to assume, according to Remark 7, that

$$
I\left(\pi^{0}, G\right) \neq 0 .
$$

\section{AKNOWLEDGMENT}

We thank C. Fabry, M. Ramos and M. Willem for stimulating discussions leading to an improvement of the proof of Theorem 1.

\section{REFERENCES}

1. V. V. Amel' 'kin, I. V. Gaishun and N. N. Ladis, Periodic solutions in the case of a constantly acting perturbation, Differential Equations 11 (1975), 1569-1573.

2. J. W. Bebernes and K. Schmitt, Periodic boundary value problems for systems of second order differential equations J. Differential Equations 13 (1973), 32-47.

3. I. Berstein and A. Halanay, The index of a critical point and the existence of periodic solutions to a system with small parameter, Dokl. Akad. Nauk 111 (1956), 923-925 (translation and remarks by G. B. Gustafson).

4. N. A. Bobylev, The construction of regular guiding functions, Soviet Math. Dokl. 9 (1968), 1353-1355.

5. A. Capietto and F. Zanolin, An existence theorem for periodic solutions in convex sets with applications, Results in Math. 14 (1988), 10-29.

6. _ A continuation theorem for the periodic BVP in flow-invariant ENRs with applications, J. Differential Equations 83 (1990), 244-276.

7. S. N. Chow and J. Mallet-Paret, The Fuller index and global Hopf bifurcation, J. Differential Equations 29 (1978), 66-85.

8. J. Cronin, The number of periodic solutions of nonautonomous systems, Duke Math. J. 27 (1960), 183-194.

9. __ Lyapunov stability and periodic solutions, Bol. Soc. Mat. Mexicana 9 (1964), 22-27.

10. __ The point at infinity and periodic solutions, J. Differential Equations 1 (1965), 156170.

11. __ Periodic solutions of some nonlinear differential equations, J. Differential Equations 3 (1967), 31-46.

12. __ Periodic solutions of nonautonomous equations, Boll. Un. Mat. Ital. (4) 6 (1972), 45-54.

13. E. N. Dancer, Boundary value problems for weakly nonlinear ordinary differential equations, Bull. Austral. Math. Soc. 15 (1976), 321-328. 
14. _ Symmetries, degree, homotopy indices and asymptotically homogeneous problems, Nonlinear Analysis, TMA 6 (1982), 667-686.

15. T. Ding, R. Iannacci and F. Zanolin, On periodic solutions of sublinear Duffing equation, J. Math. Anal. Appl. (to appear).

16. L. Fernandes and F. Zanolin, Periodic solutions of a second order differential equation with one-sided growth restrictions on the restoring term, Arch. Math. (Basel) 51 (1988), 151-163.

17. A. Fonda and P. Habets, Periodic solutions of asymptotically positively homogeneous differential equations, J. Differential Equations 81 (1989), 68-97.

18. A. Fonda and F. Zanolin, Periodic solutions to second order differential equations of Liénard type with jumping nonlinearities, Comment. Math. Univ. Carolin. 28 (1987), 33-41.

19. S. Fučik, Solvability of nonlinear equations and boundary value problems, Reidel, Dordrecht, 1980.

20. S. Fučik and V. Lovicar, Periodic solutions of the equation $x^{\prime \prime}+g(x)=p$, Časopis Pěst. Mat. 100 (1975), 160-175.

21. M. Furi and M. P. Pera, A continuation principle for forced oscillations on differentiable manifolds, Pacific J. Math. 121 (1986), 321-338.

22. R. E. Gaines and J. Mawhin, Coincidence degree and nonlinear differential equations, Lecture Notes in Math., vol. 586, Springer-Verlag, Berlin, 1977.

23. R. E. Gomory, Critical points at infinity and forced oscillations, Contributions to the Theory of Nonlinear Oscillations, vol. 3, Ann. of Math. Studies, no. 36, Princeton Univ. Press, Princeton, N. J., 1956, pp. 85-126.

24. A. Granas, The Leray-Schauder index and the fixed point theory for arbitrary ANRs, Bull. Soc. Math. France 100 (1972), 209-228.

25. A. Halanay, În legătură cu metoda parametrolui mic (Relativement à la méthode du petit paramètre), Acad. R. P. R. Bul. St. Sect. Mat. Fiz. 6 (1954), 483-488.

26. __ Solutions périodiques des systèmes non-linéaires à petit paramètre, Rend. Accad. Naz. Lincei (Cl. Sci. Fis. Mat. Natur.) (8) 22 (1957), 30-32.

27. _ Differential equations, stability, oscillations, time lags, Academic Press, New York, London, 1966.

28. J. K. Hale and A. S. Somolinos, Competition for fluctuating nutrient, J. Math. Biology 18 (1983), 255-280.

29. S. T. Hu, Theory of retracts, Wayne State Univ. Press, Detroit, Mich., 1965.

30. M. A. Krasnosel'skii, The operator of translation along the trajectories of differential equations, Transl. Math. Monographs, vol. 19, Amer. Math. Soc., Providence, R.I., 1968.

31. M. A. Krasnosel'skii and P. P. Zabreiko, Geometrical methods of nonlinear analysis, Springer-Verlag, Berlin, 1984.

32. A. Lando, Periodic solutions of nonlinear systems with forcing term, J. Differential Equations 3 (1971), 262-279.

33. $\ldots$, Forced oscillations of two-dimensional nonlinear systems, Applicable Anal. 28 (1988), 285-295.

34. A. Lasota, Une généralisation du premier théorème de Fredholm et ses applications à la théorie des équations différentielles ordinaires, Ann. Polon. Math. 18 (1966), 65-77.

35. A. Lasota and Z. Opial, Sur les solutions périodiques des équations différentielles ordinaires, Ann. Polon. Math. 16 (1964), 69-94.

36. A. C. Lazer and P. J. McKenna, A semi-Fredholm principle for periodically forced systems with homogeneous nonlinearities, Proc. Amer. Math. Soc. 106 (1989), 119-125.

37. J. Leray and J. Schauder, Topologie et equations fonctionelles, Ann. Sci. Ecole. Norm. Sup. (3) 51 (1934), 45-78.

38. W. S. Loud, Periodic solutions of $x^{\prime \prime}+c x^{\prime}+g(x)=\varepsilon f(t)$, Mem. Amer. Math. Soc., No. 31 (1959).

39. J. Mawhin, Equations intégrales et solutions périodiques des systèmes différentiels non linéaires, Acad. Roy. Belg. Bull. Cl. Sci. 55 (1969), 934-947. 
40. __ Existence of periodic solutions for higher order differential systems that are not of class D, J. Differential Equations 8 (1970), 523-530.

41. _ _ Periodic solutions of nonlinear functional differential equations, J. Differential Equations 10 (1971), 240-261.

42. __ Periodic solutions of some perturbed differential systems, Boll. Un. Mat. Ital. (4) 11 (1975), 299-305.

43. __ Topological degree methods in nonlinear boundary value problems, CBMS Regional Conf. Ser. Math., no. 40, Amer. Math. Soc., Providence, R.I., 1979.

44. __ Point fixes, point critiques et problemes aux limites, Séminaire de Mathématiques Supérieures, vol. 92, Les Presses de l'Université de Montréal, 1985.

45. __ A simple proof of a semi-Fredholm principle for periodically forced systems with homogeneous nonlinearities, Arch. Math. (Brno) 25 (1989), 235-238.

46. J. Mawhin and J. R. Ward, Periodic solutions of some forced Liénard differential equations at resonance, Arch. Math. (Basel) 41 (1983), 337-351.

47. E. Muhamadiev, Construction of a correct guiding function for a system of differential equations, Soviet Math. Dokl. 11 (1970), 202-205.

48. _ On the theory of periodic solutions of systems of ordinary differential equations, Soviet Math. Dokl. 11 (1970), 1236-1239.

49. M. Nagumo, Über die Lage der Integralkurven gewöhnlicher Differentialgleichungen, Proc. Phys.-Math. Soc. Japan (3) 24 (1942), 551-559.

50. R. D. Nussbaum, The fixed point index and some applications, Séminaire de Mathématiques Supérieures, vol. 94, Les Presses de l'Université de Montréal, 1987.

51. P. Omari, Gabriele Villari and F. Zanolin, Periodic solutions of the Liénard equation with one-sided growth restrictions, J. Differential Equations 67 (1987), 278-293.

52. P. Omari and F. Zanolin, On forced nonlinear oscillations in $n$-th order differential systems with geometric conditions, Nonlinear Analysis, TMA 8 (1984), 723-784.

53. Z. Opial, Sur les solutions périodiques de l'équation différentielle $x^{\prime \prime}+g(x)=p(t)$, Bull. Acad. Polon. Sci. Sér. Sci. Math. Astronom. Phys. 8 (1960), 151-156.

54. J. Palis, Jr. and W. De Melo, Geometric theory of dynamical systems, Springer-Verlag, Berlin, 1982.

55. J. Pejsachowicz and A. Vignoli, On the topological coincidence degree for perturbations of Fredholm operators, Boll. Un. Mat. Ital. (5) 17-B (1980), 1457-1466.

56. V. A. Pliss, Nonlocal problems of the theory of oscillations, Academic Press, New York, 1966.

57. R. Reissig, Periodic solutions of a nonlinear $n$-th order vector differential equation, Ann. Mat. Pura Appl. (4) 87 (1970), 111-123.

58. R. Reissig, G. Sansone and R. Conti, Qualitative Theorie nichtlinearer Differentialgleichungen, Cremonese, Roma, 1963.

59. N. Rouche and J. Mawhin, Ordinary differential equations, Pitman, London, 1980.

60. K. Schmitt, Periodic solutions of small period of systems of $n$-th order differential equations, Proc. Amer. Math. Soc. 36 (1972), 459-463.

61. R. Srzednicki, On rest points of dynamical systems, Fund. Math. 126 (1985), 69-81.

62. _ Periodic and constant solutions via topological principle of Wazewski, Acta Math. Univ. Iag. 26 (1987), 183-190.

63. F. Stoppelli, Su un'equazione differenziale della meccanica dei fili, Rend. Accad. Sci. Fis. Mat. Napoli 19 (1952), 109-114.

64. M. Urabe, Nonlinear autonomous oscillations. Analytical theory, Academic Press, New York, 1967.

65. P. Volkmann, Zur Definition des Koinzidenzgrades, preprint, 1981. 
66. J. R. Ward, Conley index and non-autonomous ordinary differential equations, Results in Math. 14 (1988), 191-209.

67. J. A. Yorke, Invariance for ordinary differential equations, Math. Systems Theory 1 (1967), 353-372.

International School for Advanced Studies, Strada Costiera 11, 34014 Trieste, Italy Current address: Department of Mathematics, University of Torino, Via Carlo Alberto, 10, 10123 Torino, Italy

Universite de Louvain, Institut Mathematique, Chemin du Cyclotron 2, B-1348 LOUVAIN-LA-NEUVE, BELGIUM

Department of Mathematics and Computer Sciences, University of Udine, Via Zanon 6, 33100 Udine, ITALY 